\title{
An Investigation Into the Determinants of Flight Cancellations*
}

\author{
Nicholas G. Rupp ${ }^{\dagger}$ \\ Department of Economics \\ East Carolina University \\ Greenville, NC 27858-4353 \\ George M. Holmes ${ }^{\ddagger}$ \\ University of North Carolina \\ 725 Airport Road, CB 7590 \\ Chapel Hill, NC 27599-7590
}

\begin{abstract}
Keywords: Service Quality, Flight Cancellations, Airlines
JEL Classification: L13, L93.
\end{abstract}

June 22, 2005

\begin{abstract}
This paper uses Bureau of Transportation data on 35 million domestic flights between 1995 and 2001 to investigate the determinants of flight cancellations. This paper is novel in two regards since it focuses exclusively on flight cancellations and it explores the service quality and flight revenue relationship. We find that carriers have some control over the occurrence of flight cancellations given that cancellations are significantly less likely on Thursday, Friday, and Sunday and for the last flight of day. There is some evidence that links cancellations with revenue.
\end{abstract}

\footnotetext{
${ }^{*}$ We thank Joe Hopkins, Harumi Ito, Darin Lee, Chris Mayer, Ed Schumacher, Lester Zeager, several anonymous airline operations employees, seminar participants at Appalachian State University, East Carolina University, International Industrial Organization Conference, and Southern Economic Association Conference, and two referees for their helpful comments. Sherrine Ahmed and Jim Goodman provided research assistance.

${ }^{\dagger}$ Contact at ruppn@mail.ecu.edu or (252) 328-6821.

‡Contact at mark_holmes@unc.edu.
} 
"Canceling a flight for economic reasons was totally taboo before, but we're seeing it now."

(Robert Harrell, Vice President for American Express Travel Related Services, Wall Street Journal, 15 February 1991.)

\section{Introduction}

U.S. airline carriers had 665.5 million enplanements in 2000 , which generated $\$ 93.6$ billion in passenger revenue and employed 679,967 individuals (Air Transport Association 2001 Annual Report). Yet airlines have historically provided poor customer service: approximately one in four flights were delayed, canceled, or diverted between 1995 and 2002 (see Table 1). The most common consumer air travel complaint in 2000 was flight problems (e.g., cancellations, delays, and missed connections), an increase of 30 percent from 1999 (Air Travel Consumer Report, February 2001, p. 34), while revenue passenger miles increased only 6 percent over the same period. ${ }^{1}$ Consumer frustration with airlines was typified by a Chicago businessman, who filed suit against United Airlines in 2000, seeking financial damages to cover ticket refunds, reimbursements for alternative transportation, compensation for emotional distress, lost vacation days, and missed business opportunities following a rash of cancellations due to unresolved contractual issues with United pilots (Carey, 2000). In 1999, due to poor airline service quality, U.S. Congressional hearings debated the merits of a passenger bill of rights. Ultimately, fourteen major domestic airlines voluntarily agreed in 1999 to the Airline Customer Service Commitment, which promised improved treatment of air travelers. In a follow-up study one year later, the U.S. Department of Transportation found that airlines were falling short of their promises due to extensive flight delays and long check-in lines (Heller, 2000).

This paper examines flight cancellations by using a sample of U.S. Bureau of Transportation Statistics (BTS) data for 35 million domestic flights in the U.S. by major carriers between January 1995 and August 2001. During this period there were more than one million flight cancellations. Moreover, cancellations became more frequent, increasing from 91,905 (in 1995) to more than twice that amount, 231,198 flights (in 2001), which corresponds to a cancellation rate of 1.73 and 2.79 percent, respectively, of all scheduled domestic flights. This study is novel in two aspects. First, it is one of the first to investigate the deter- 
minants of flight cancellations. ${ }^{2}$ Second, the paper explores the relationship between service quality and revenues during normal daily operations.

Related theoretical work on flight cancellations is provided by networking models, which determine the optimal aircraft recovery schedule following a hub closure (Yan and Yang, 1996; Thengvall et al. 2000; Thengvall et al. 2001). Previous empirical research on service quality primarily has focused on flight delays, instead of cancellations (e.g., Mayer and Sinai, 2003a \& 2003b; Mazzeo, 2003).

In the first six months after the BTS began tracking causes of flight delays in August, 2003, the four most common delay causes (in order of relevance) are late arriving aircraft, air carrier delay, national aviation system delay, and extreme weather. ${ }^{3}$ Hence this paper controls for each of these measures and we find many significant non-weather cancellation factors matter such as day of the week, departure time, and number of daily scheduled flights on a route. An analysis of flight cancellations is beneficial given that (i) cancellations are more inconvenient for passengers than flight delays (e.g., following a cancellation passengers who are fortunate enough to be re-booked on the carrier's next scheduled departure on the route wait an average of over five hours, whereas the typical delayed flight departs an average of 52 minutes late); (ii) airline performance is a high-priority issue for travelers, airlines, and lawmakers; and (iii) recent consolidation (American Airlines acquired TWA in 2001), bankruptcy filings (US Airways and United Airlines declared bankruptcy in 2002), and scheduling cut-backs since the September 11th terrorist attacks (Carey and McCartney, 2001) may affect cancellations. Hence this paper investigates the link between flight cancellations and competition at both the route and airport level.

This paper examines four potential flight operation objectives originally proposed by Rupp et al. (2005), that carriers might pursue. There are a number of different competing objectives that carriers could follow in making daily flight operation decisions in order to maintain existing flight schedules. First, route and airport competition may motivate carriers to avoid lapses in service quality by limiting cancellations. Second, carriers may attempt to avoid disruption of their network operations by limiting the cancellation of flights to and from their hub airports. Third, carriers may choose to limit the number of passengers displaced by a service interruption by conducting fewer cancellations of larger and fuller planes. Fourth, carriers may protect their profits on high revenue routes by attempting to provide high service quality 
(fewer cancellations) on such routes.

We examine the effect of four classes of explanatory variables on flight cancellations. Following the previously cited literature, our regressions include economic, competitive, logistical, and weather measures at the route, airport, and aircraft level. We find evidence which suggests that route competition improves service quality. At the airport level, however, flight cancellations are independent of airport concentration. We find significantly fewer flight cancellations at hub airports, which highlights the importance of hub flights in maintaining a flight network. There is considerable support for the hypothesis that carriers minimize passenger inconvenience. We present some empirical evidence that links revenue with flight cancellations. In the next section, we examine four potential flight operation objectives. We then describe the econometric specification used in the empirical analysis. The data are discussed in section 3 , and findings are presented in section 4. We conclude the paper with some public policy implications for flight cancellations.

\section{$2 \quad$ Flight Operation Objectives}

We envision two types of cancellations. First, "stochastic cancels" are situations where the short run supply of available aircraft is exogenously decreased (e.g., severe weather limits airport operations, or an equipment failure/maintenance repair is necessary before the aircraft is operational). Second, "strategic cancellations" are situations in which the airline cancels a flight for strictly economic reasons (e.g., low passenger bookings). Note that economic factors can still play an important role even when stochastic cancels occur since airlines may prioritize high-profit flights over low-profit flights.

\subsection{Competition}

Kranton (2003) presents a model where price competition in a market can eliminate the incentive for firms to produce a high-quality good when consumers cannot observe the quality of the good prior to purchase. Empirical research in a variety of industries such as public schools (Hoxby, 2000), health care (Kessler and McClellan, 2000) and surface freight transportation (Ellig and Kelley, 2002) have found that competition improves service quality, yet the competitive effects on service quality in the airline industry 
remain unclear. Mazzeo (2003) finds more competitive routes have better service quality in terms of less frequent and shorter flight delays. ${ }^{4}$ Mayer and Sinai (2003b), however, report the opposite result that less competitive routes provide better service. Oum et al. (2000) find mixed evidence of how competition affects service quality in their examination of global airline alliances and international flight delays. At the airport level, Brueckner (2002) and Mayer and Sinai (2003a) find more concentrated (less competitive) airports have fewer flight delays. ${ }^{5}$ Given that the competitive effects on airline service quality are ambiguous, this paper hopes to provide some insight into how one measure of airline service, flight cancellations, is affected by route competition.

We explore three different variables to characterize route competition. First, since more than half of the sample involves routes served by a single carrier, we examine monopolist routes to see if less competitive routes have worse service quality. Second, market share, the proportion of daily scheduled flights on route $r$ provided by carrier $j$, provides a continuous measure of route-level competition. Finally, we track the performance of routes served by two carriers to determine if performance differs for large and small duopolists. Following Mayer and Sinai (2003b), the carrier with a larger (smaller) route market share is the large (small) duopoly carrier. Hence the first potential operations objective is that carriers provide better service quality on competitive routes in order to retain passengers.

\section{$2.2 \quad$ Flight Network}

The second hypothesis suggests that carriers give priority to hub origination and destination flights to maintain their flight network. We define airline hub origination (destination) as carriers with 26 or more connections at the origination (destination) airport. By not canceling hub origination flights, carriers can keep their network intact. In addition, hub origination flights may have fewer cancellations than non-hub flights due to some supply-side issues. Maintenance and/or staffing issues can be more easily addressed for flights originating at a carrier's hub airport due to the greater availability of spare parts, replacement aircraft, mechanics, ground personnel, and flight crews. For example, Northwest Airlines has pilot bases and flight attendant bases at all three of its U.S. hub airports (Memphis, Minneapolis/St. Paul, and Detroit) and Northwest operates two U.S. maintenance bases in Minneapolis and Duluth, MN. 
Hub destination flights may have fewer flight cancellations than non-hub destination flights due to demand side issues - carriers need these flights to arrive in order to keep their network intact and to enable connecting passengers to reach their next flight. Morrison and Winston (1995, p. 44) report that at a typical hub a majority of passengers make connections. In addition, flights destined for hubs are more likely to haul passengers making international connections. Canceling a hub destination flight is more inconvenient for both domestic and international connecting passengers. Discussions with flight operation employees of mainline U.S. carriers confirm that carriers are concerned about getting passengers to international destinations.

We further explore this flight network hypothesis by distinguishing the size of an airline's hub. Similar to Mayer and Sinai (2003a), we define airline hub sizes by the number of connecting flights at the origination airport, such that airlines having 71 or more connections are large airline hub origination, between 46 and 70 connections medium airline hub origination, and between 26 and 45 connections small airline hub origination. Hub airline operations at destination airports are defined in a similar manner. We expect that the flight network effect is most pronounced for large airline hub origination \& destination.

\subsection{Passenger Inconvenience}

The third hypothesis is that carriers attempt to limit passenger inconvenience from service disruptions. Limiting inconvenience is related to the fourth hypothesized objective (revenue maximization) since the number of passengers is also a component in the revenue calculation. We use four variables to measure the magnitude of passenger inconvenience - additional planes needed, seating capacity, daily scheduled flights, and last flight of day. First, the additional planes needed to accommodate displaced passengers after a flight cancellation is defined as: load factor/(1-load factor), where load factor is the monthly average proportion of total seats that are occupied by passengers for carrier $\mathrm{j}$ on route $\mathrm{r}$. For example, if a route has an average load factor of 0.75 , then additional planes needed equals $0.75 / 0.25=3$, where 3 is the approximate number of additional planes needed to accommodate displaced passengers. ${ }^{6}$ Carriers can limit passenger inconvenience by canceling flights on routes in which fewer additional planes are needed. Second, if carriers are limiting passenger inconvenience, then we should find fewer cancellations for planes with larger seating 
capacity. Third, canceling a flight on a route with frequent daily service minimizes passenger inconvenience since frequently served routes have shorter waiting periods until the next scheduled departure. Finally, carriers can also reduce passenger inconvenience by not canceling the last flight of the day for carrier $j$ on route $r .^{7}$ Getting the last flight to its destination also avoids some costly alternatives such as issuing refunds, rebooking passengers on a competitor's flight, and/or paying for overnight accommodations for displaced passengers. ${ }^{8}$

\subsection{Revenue Maximization}

Neoclassical economic theory assumes that airlines maximize profits. Since route-level profitability figures are unavailable and costs are likely more constant across routes than revenue, we use route revenue as a profit proxy. There are likely to be both short-term and long-term aspects of the relationship between revenue and service quality. In the short-term carriers may avoid canceling a high revenue flight in order to avert costly passenger reimbursements to those who abort their trips due to service disruptions. ${ }^{9}$ If the theoretical switching model, proposed by Suzuki (2000) and calibrated with aggregate US DOT data, is accurate and passengers who experience poor service quality are more likely to switch carriers, then the effect of a flight cancellation may also be felt long-term by the carrier. This may be especially true if travelers blame flight cancellations on the carrier rather than a problem that is beyond the carrier's control (i.e., severe weather). Hence our fourth and final hypothesis is that carriers provide better service quality on more profitable (high revenue) routes.

We consider three revenue measures including average revenue (quarterly average one-way passenger fare multiplied by the monthly average number of occupied seats on the plane), potential revenue (quarterly average one-way passenger fare multiplied by seating capacity of the plane), and yield (dollars per revenue passenger mile). This paper is one of the first to link route revenue with flight service quality. Rupp et al. (2005) find that higher potential revenue flights receive better service quality (fewer cancellations and delays) after an airport reopens following a security-related airport closure. During irregular operations, potential revenue may be the preferred revenue measure since every seat is likely to be taken after an airport reopens due to the large number of flight cancellations. During normal operations, however, average revenue 
may be a better measure of lost revenue from a flight cancellation since average revenue does not assume that all seats are occupied. Instead, this revenue measure assumes that the flight has the monthly average passenger load. Although our examination of the revenue and service quality relationship is novel, previous studies on airline prices have used yield (Windle and Dresner, 1999; Lee and Ito, 2004). Given that average fares are higher for longer flights, all estimations control for flight length by including non-stop distance blocks between airports for short flight (<400 miles) and middle distance (400 to 800 miles) flights.

\subsection{Econometric Specification}

We now specify the empirical model. Due to the presence of a discrete dependent variable (flight cancellation), we employ a probit model to estimate our parameters of interest:

$$
L_{i t}=\left[\Phi\left(X_{i t} \beta\right)\right]^{z_{i t}}\left[1-\Phi\left(X_{i t} \beta\right)\right]^{1-z_{i t}}
$$

where $z_{i t}=1$ if the $i^{t h}$ flight on day $t$ is canceled and 0 otherwise and $X_{i t}$ is a vector of carrier, route, airport, weather, and time period characteristics. In addition, for a baseline of comparison with previous flight delay studies, we present one estimation for the occurrence of a flight departure delay. Hence $z_{i t}=1$ if the $i^{\text {th }}$ flight on day $t$ departs more than 15 minutes after its scheduled departure time and 0 otherwise.

Given that we consider five different route competition measures, we compare the results of the models using binary tests for model selection due to Davidson and MacKinnon (1993) and Vuong's (1989) nonnested test. ${ }^{10}$ Results of these tests, along with the comparisons of log-likelihood values, suggest that models using monopoly as the route competition measure are better specified. Hence monopoly is the default variable for route competition.

All flight cancellation and delay models control for individual carrier, month, year, and day of week effects by using indicator variables. ${ }^{11}$ We do not present the estimated effects for day of week, month, and specific carriers because they are intended as control variables, not as independent variables interesting

on their own. Nonetheless, the appendix reports coefficients, standard errors, and marginal effects for these indicator variables for three estimated flight cancellation models reported on Table 3 which are 
representative of the indicator variable effects.

A limitation of this research is that we only observe the flight outcome (cancel or not cancel). A carrier, however, makes additional choices and can update these decisions before the flight departs, all of these decisions will influence the likelihood of a cancellation. For example, airlines can choose both the type and size of an aircraft serving a route, hence carriers have considerable influence on load factor and total passengers for each route. In addition, in the winter, the carrier may opt to direct larger planes through its good weather hub and smaller planes through the hub with worse weather. Our weather variables control for severe weather days, however, the point remains that carriers have considerable influence over some of our explanatory variables. We also note that while the regressions do not account for every different model or type of aircraft, the estimations in model 6 do account for aircraft manufacturer and aircraft age, two potentially important choice variables.

\subsection{Correlation Issues}

We are concerned about correlation between the unobserved terms in three dimensions. First, intertemporal correlation within a route may exist. For example, airport-specific shocks (such as less experienced employees or airport construction) may lead to this condition. Second, a similar argument would suggest correlation across carriers within the same route. Furthermore, airlines may be more willing to offer poor service on a route if the competition also offers poor service on the same route. Third, correlation within carrier across "opposite" routes may exist. That is, if a flight from airport A to airport B is canceled, then the flight from B to A may also be cancelled. The prototypical example is if a flight from a hub to an outlying airport is canceled, then there is no aircraft in the outlying airport, so the return flight is canceled by default.

We deal with the first two correlation types by estimating standard errors via block bootstrapping (Härdle et al. 2002). We find that the bootstrapped standard errors are, on average, approximately $50 \%$ larger than the standard errors assuming independence. We deal with the third correlation issue by randomly selecting flights in one direction only, either "A to B" or "B to A", but not both. 


\section{The Data}

\subsection{The Sample}

Our data and a majority of the variables are constructed from individual flights obtained from the U.S. Bureau of Transportation Statistics (BTS) TranStats database (www.transtats.bts.gov). Airlines with at least 1 percent of domestic scheduled passenger revenues are required to submit monthly performance reports to the BTS. Hence these flight data cover all nonstop scheduled-service domestic flights by the ten largest mainline or "major" U.S. carriers, ${ }^{12}$ which account for more than 85 percent of domestic revenues in 2000 (Air Travel Consumer Report, January 2001). The ten major carriers are required to report flight operations in 29 U.S. airports. Beginning in 1995, every major airline has voluntarily reported all domestic operations to the BTS. Prior to 1995, carriers did not report flight delays or cancellations due to mechanical difficulties. The result is the best source of airline cancellation rates and on-time performance data. Covering the period 1995-2002, Table 1 summarizes annual flight operations. The table shows that the percentage of late arrivals has generally increased since 1995, reaching a peak in 2000 of 27 percent, and a dramatic reduction in flight delays in 2002. Cancellation rates exhibit a similar pattern, increasing each year between 1997 and 2001 with the highest cancellation rate of 3.3 percent occurring in 2000. More recently, cancellation rates have fallen in 2001 and 2002. The percentage of flights diverted has remained relatively constant, around 0.2 percent. Finally, on-time performance improved substantially in 2002 with on-time arrival rates exceeding 80 percent and flight cancellations dropping to 1.2 percent.

[Place Table 1 about here]

We obtained every domestic flight in the U.S. between January 1995 and August 2001 from the BTS TranStats database. We restrict our attention to the pre-September 11th period since we expect a massive structural shift around this period (rendering pooling unappealing) and more data is available in the preperiod than the post-period. ${ }^{13}$ Since there are approximately 35 million individual flights during this period, to obtain a more manageable data set we are forced to reduce the sample size. As mentioned previously, the sample is reduced by $50 \%$ to avoid correlation between routes serving the same two airports. Specifically, 
the sample only includes flights in one direction, either from airport A to airport B or from B to A. We also omit days in which a carrier cancels more than $95 \%$ of their scheduled domestic daily flights. This criterion drops days in which a labor strike prohibits flight operations. Next, we randomly select ten percent of the remaining flights, resulting in a sample size of 1,447,096 individual flights.

All variables are constructed from the original data set, with only the estimations using the smaller randomly selected sample. Individual flight data provide efficiency gains over monthly aggregate cancellation data if the individual flight characteristics (e.g., departure time or daily precipitation), contribute to the cancellation rate. Daily flight data enable us to control for day of the week effects. In fact, we find significantly fewer flight cancellations for weekend flights (Thursday, Friday, and Sunday) compared to Wednesday flights (see Appendix). Friday and Sunday are busy travel days given the surge of leisure travelers on the weekends. Hence canceling such flights would be especially costly for carriers and inconvenient for passengers. The Sunday marginal effect of -0.0055 from model (1) suggests that the cancellation rate for Sunday is 0.55 percentage points lower than Wednesday. This percentage point reduction corresponds to Sunday flights being approximately 21 percent less likely to be cancelled, compared to Wednesday flights, given that the average flight cancellation rate in the sample is 2.56 percent.

The differences in the day of the week flight cancellation rates are not merely an artifact of varying aircraft utilization rates among the days. We find little variation in the number of scheduled weekday flights and a reduction in flight offerings on the weekend. Specifically, during the first eight months of 2001, the average number of daily scheduled U.S. domestic flights during a weekday varied little ranging from a low of 17,081 (Wednesday) to a high of 17,565 (Monday). Flight offerings are lower on Saturdays and Sundays by $14 \%$ and $5 \%$, respectively when compared to the average number of weekday flights $(17,222)$. If cancellation rates were a function of aircraft utilization rates, then we should observe the lowest cancellation rates on Saturday and little difference among the weekday cancellation rates. The results in the Appendix suggest otherwise. In sum, these results suggest that flight cancellations may not be entirely random. Consequently, all estimations include day-of-the-week indicators to control for important day of the week effects. 
Descriptive statistics for the sample appear in Table 2. We separate flights into one of four categories: non-hub airport pairs, hub to non-hub (or hub-spoke), non-hub to hub (or spoke-hub), and hub to hub flights. Recall that a carrier must offer $26+$ connecting flights at the airport to receive the "hub" designation. An examination of every domestic flight for all U.S. carriers in May 2001 reveals that hub-spoke and spoke-hub flights each comprise $37 \%$ of the sample. Non-hub airport pairs and hub-hub flights encompass $21 \%$ and $5 \%$ of the sample, respectively. The non-hub airport pairs are not entirely comprised of Southwest Airlines flights. In fact, approximately half of the flights between non-hub airports are from network carriers. We find that non-hub to non-hub flights (in comparison to all other flights) are typically shorter distance, lower average fare, slightly smaller aircraft, and have lower load factor. In addition, we find some of the lowest cancellation rates occur for these non-hub airport pairs.

[Place Table 2 about here]

Carriers provide an average of seven non-stop daily scheduled flights on a route. Five of every six flights are on routes with limited competition. Specifically, monopoly and duopoly routes comprise 53 and 30 percent of the sample, respectively. One in nine flights (11 percent) originate from a slot-controlled airport (i.e., New York LaGuardia (LGA), New York JFK, Washington Reagan National (DCA), and Chicago O'Hare (ORD)). A 'slot' provides the carrier with a short window (typically 60 or 90 minutes) in which an aircraft is allowed to land or take-off. Slots cannot be transferred nor can they be saved (i.e., the carrier either uses the slot or loses it).

We match the tail numbers provided by the BTS to the FAA Aircraft Registry database, which enables us to obtain aircraft characteristics such as seating capacity (the number of available seats on the aircraft), manufacturer (i.e., McDonnell-Douglas, Airbus, etc.), and aircraft age (the number of years since the aircraft was manufactured). Since we are unable to match tail numbers to the FAA registry database for approximately half of the sample flights, ${ }^{14}$ only one estimation (see model 5) includes aircraft specific characteristics.

We also match individual flights to quarterly passenger fare data from the Airline Origin and Destination (OEBD) Survey, which is a $10 \%$ sample of airline tickets from reporting carriers, collected by the 
U.S. Office of Airline Information of the Bureau of Transportation Statistics and included in the TranStats database. These fare data enable us to estimate the revenue of a particular flight. The sample average fare of $\$ 166$ reflects the nominal quarterly average one-way airfare for carrier $j$ on route $r$. For round-trip itineraries, the total ticket price is divided by two to obtain the one-way airfare. Average revenue is found by multiplying average fare by the monthly average number of occupied seats for carrier $j$ on route $r$, which is obtained from the Air Carrier Statistics database (also known as T-100 data bank and available at TranStats). The typical plane size of 162 seats has a potential revenue per flight of approximately $\$ 31,000$. Since the average load is two-thirds of capacity, the average revenue per flight is about $\$ 21,000$. Finally, yield is the carrier's total quarterly revenue on route $r$ divided by the total quarterly revenue passenger miles on route $r$. One caveat regarding our revenue measures is that the O\&SD Survey data only provide the average fare for a given quarter (monthly for load factor); hence we do not observe average fares paid on a particular day and flight. For example, within the same day there may be considerable variation in load factor and average fares due to differences in the scheduled departure time. Nonetheless, in these data, quarterly revenue measures and monthly capacity observations provide the smallest possible level of aggregation.

We obtain load factor from the TranStats database, which includes monthly T-100 domestic market data. This variable is the monthly average proportion of total seats that were occupied by passengers for carrier $j$ on route $r$. Load factor is multiplied by the seating capacity of the aircraft in order to obtain the monthly average number of occupied seats for the carrier. To account for the occurrence of cascading delays during the day (Mazzeo, 2003), we include a continuous time measure, time01 which renormalizes the scheduled departure time to between 0 (midnight) and 1 (23:59). Time01 and day of the week can partially, but not perfectly, control for these within day and between day differences.

Since most airports are active weather reporting stations, ${ }^{15}$ we obtain daily weather data at origination and destination airports from the U.S. National Oceanic \& Atmospheric Administration (NOAA). These weather measures include temperature, rain, and freezing rain. Minimum temperature origination (destination) is the minimum daily temperature (in Fahrenheit) at the origination (destination) airport. Rain origination (destination) is the amount of daily precipitation (in hundredths of an inch) at the origina- 
tion (destination) airport. Finally, frozen precipitation origination (destination) is daily rain origination (destination) multiplied by one if daily minimum temperature $<33$, otherwise zero. ${ }^{16}$

The sample selection criteria drops days in which a carrier cancels $95 \%$ or more of its scheduled flights. Since labor issues may cause a carrier to cancel a portion of its flights, we also use the ProQuest database to search the Wall Street Journal for keyword combinations of airline (or carrier) and labor (or strike or slow-down or sick-out or work-to-rules or CHAOS). Hence labor unrest is a binary variable, coded as one for the month in which the Wall Street Journal reports that a carrier is experiencing a previously mentioned labor issue and zero otherwise.

Since airport congestion is an important flight delay determinant (Mayer and Sinai, 2003a; Mazzeo, 2003), we include daily airport operations origination (destination), which is the total number of daily take-offs and landings at the origination (destination) airport. We also include monthly indicators in all estimations to control for both demand and seasonal fluctuations in cancellations which typically peak in January for cold weather airports.

\section{Results}

\subsection{Which Flights are Canceled?}

This paper examines various route and airport competition measures, aircraft characteristics, flight revenues, and airport size effects by estimating fourteen flight cancellation models and one flight delay model. The latter enables a comparison with the flight delay literature. All estimated models include carrier, day of the week, month, and year indicator variables. We also report marginal effects in Tables 3-8, which are defined as the effect on the probability that the average flight is canceled. We begin by examining the first hypothesis which proposes that route competition improves service quality.

\subsubsection{Route Competition}

Most results are consistent with this first hypothesis since three different route competition measures on

Table 3 all reveal a similar result that competitive routes have fewer flight cancellations. ${ }^{17}$ Model (1) 
indicates that monopoly routes have significantly more cancellations. This result is robust across a variety of specifications since monopoly registers statistical significance in seven of nine estimated cancellation models which exclude airport fixed effects. The marginal effects in model (1) are interpreted as follows: monopoly routes have 0.23 percentage points higher cancellation rates which corresponds roughly to a $9 \%$ increase in flight cancellations. In other words, for a carrier that has seven daily scheduled flights on a route (or 2,555 scheduled flights a year), we estimate that a monopolist carrier would cancel an additional six flights per year. In addition to more flight cancellations, we also find that monopoly routes have significantly more departure delays (see Model 7). This latter result is also consistent with a delay study by Mazzeo (2003). ${ }^{18}$

[Place Table 3 about here]

Higher flight cancellation rates for monopoly routes is especially interesting given that Borenstein and Netz (1999) find that monopolist carriers have easier to maintain flight schedules due to greater departure time differentiation which enables a monopolist to avoid peak travel congestion times. Borenstein and Netz report that routes served by multiple carriers, which operate the same total number of flights, have flight schedules with less departure-time differentiation (i.e., grouping departures around peak travel times) compared to a monopolist which operates the same number of flights. Despite these easier to satisfy flight schedules, we find that monopoly carriers have significantly more flight cancellations.

Model (2) reveals marginally higher (significant at the $10 \%$ level) cancellation rates for carriers with higher route level market share. This finding of worse service quality for carriers with greater market share is consistent with the previously discussed monopoly results. Model (3) examines the performance of both monopoly and duopoly carriers. We find that monopoly and small duopoly carriers have more cancellations. Surprisingly, large duopoly carriers are neither more likely nor less likely to cancel flights. Given the similar results for these three route competition measures and since a majority of routes (53\%) are served by monopoly carriers, the paper uses monopoly as the default measure of route competition. We also note that improvements in service quality from competition are limited to route competition and do not extend to airport competition. ${ }^{19}$ 
Since monopoly routes are more prevalent at smaller airports, ${ }^{20}$ the increase in flight cancellations maybe driven by an airport effect (i.e., lack of mechanics at small airports) rather than a monopoly effect. To control for airport effects, model (5) includes dummy variables for each origination and destination airport. We find that the monopoly coefficient retains its positive sign, however, loses its statistical significance. This finding indicates that monopoly routes are experiencing more cancellations due to an airport effect rather than a monopoly effect. Finally, we should note that we find few notable changes in the airport fixed effects estimation (comparing models $4 \& 5$ on Table 4). This suggests that our results (other than monopoly) appear robust to this alternative specification. In sum, the estimations that exclude airport fixed effects indicate that competitive routes have lower flight cancellation rates. Once we control for airport specific effects, however, the link between monopoly routes and flight cancellations dissipates. Next, we turn our attention to the second hypothesis that carriers provide better service to and from their hub airports in order to maintain their flight network.

\subsubsection{Flight Network}

The estimated cancellation models reveal a consistent and overwhelming result that both airline hub origination and destination flights have significantly fewer cancellations. These airline hub effects are nontrivial. For example, model (1) indicates that cancellation rates are $28 \%$ lower for airline hub origination flights and $26 \%$ lower for airline hub destination flights. We attribute these results to the previously discussed demand and supply side issues. Carriers want to avoid canceling flights originating from their hubs to keep their flight network operating. The supply-side issues for fewer airline hub origination cancellations include better access to maintenance facilities, ground personnel, replacement flight crews, and spare parts.

On the other hand, demand-side issues arise for fewer airline hub destination cancellations since carriers need these aircraft to arrive at the hub in order to maintain their flight network. An examination of late night scheduled departures (between 10 p.m. and 11 p.m.) reveals that cancellation rates are $26 \%$ lower for airline hub destination flights. ${ }^{21}$ This finding suggests that carriers are positioning themselves for normal hub operations the following day. In addition, airline hub destinations typically involve con-

necting passengers some of whom may be bound for an international destination. Hence canceling an 
airline hub destination flight is inconvenient for connecting passengers, and especially so for international bound travelers. We should also note that better service (i.e., fewer cancellations) for hub airlines is a stark contrast to the flight delay literature (Mayer and Sinai, 2003a \& 2003b; Mazzeo, 2003) which has extensively documented worse hub service (i.e., both more frequent and longer delays) for hub origination and destination flights.

To further explore this flight network hypothesis, model (4) examines the size of an airline's hub. If this network hypothesis holds, then carriers should provide better service at larger airline hubs. In fact, we find that flight cancellations monotonically decrease as the size of a carrier's hub increases for both origination and destination airports. For example, the marginal effects for large (-0.011), medium (-0.010), and small (-0.004) airline hub originations correspond to a reduction in the flight cancellation rate of $41 \%, 37 \%$, and $16 \%$ respectively. Once again, the better performance at larger hub airports is likely due to larger hubs having more available resources.

\section{[Place Table 4 about here]}

We also find fewer flight cancellations at larger airline hub destinations. Specifically, large, medium, and small airline hub destinations have $40 \%, 33 \%$, and $27 \%$ respectively, lower flight cancellation rates. Our discussions with airline operations personnel revealed that airlines have a keen interest in the welfare of international travelers. Hence we attribute the better performance for hub-bound flights to more international travelers making connections. For example, in May 2001, large hub destination airports had an average of 33.67 scheduled daily international departures, while medium and small hub destinations had 14.19 and 10.10 daily international departures, respectively. In comparison, non-hub destinations offered an average of 0.21 daily international departures during this same time period.

Since carriers can choose the location of their hub airport, we expect hub airports to have more favorable weather conditions. While all regressions control for daily weather conditions at both origination and destination airports, nonetheless model (5) includes airport dummy variables to control for airport specific effects. We find that all hub airline size variables maintain their statistical significance. Once again the larger hubs have the largest impact on reducing flight cancellations. We should note that the inclusion of 
airport fixed effects does reduce the magnitude of the airline hub coefficients and marginal effects. In sum, there is overwhelming evidence to support the second hypothesis that carriers seek to maintain their flight networks. Next, we turn our attention to the third hypothesis that carriers limit passenger inconvenience.

\subsubsection{Passenger Inconvenience}

There is considerable evidence which suggests that carriers minimize passenger inconvenience by not canceling fuller planes, infrequently served routes, and the final flight of the day. Moreover, these results are robust across various modeling specifications. Specifically, every estimated model indicates that cancellations are less common on routes that require more additional planes needed to transport displaced passengers following a flight cancellation. This nonlinear measure of seat occupancy rates shows that fuller planes have fewer cancellations. ${ }^{22}$ Fewer cancellations for planes with higher occupancy rates does come at a cost, since model (7) suggests that routes with fuller planes take longer to load and hence experience more frequent flight delays, a result consistent with Rupp et al. (2005). In sum, we find that full planes are more likely to be delayed yet less likely to be canceled.

Carriers that offer more daily scheduled flights on a route are more likely to cancel a flight. We do not, however, attribute this result to congestion since the daily total airport operations at both origination and destination are co-variates and should control for airport congestion. Instead, we believe that canceling a flight on a route with frequent daily service limits passenger inconvenience since carriers have more opportunities to accommodate displaced passengers and hence minimize the waiting time until the next flight. Shorter waiting periods also discourage passengers from making the costly demand of being rebooked on another carrier. Using the estimated parameters from model (1), figure (1) graphs both the predicted and actual probability of a flight cancellation as a carrier schedules more daily flights on a route. This figure reveals that as the number of daily scheduled flights increases, holding all other characteristics constant, the probability of a flight cancellation also increases. Restricting the graph to routes in which a carrier schedules between 0 and 16 daily flights (95 percent of all flights in our sample are on routes with 16 or fewer daily flights), we find that the predicted cancellation rates mirror the actual rates.

[Place Figure 1 about here] 
Model (6) controls for a variety of aircraft characteristics including: age, number of seats, and manufacturer. We find significantly fewer cancellations for younger aircraft, while the estimates reveal no link between cancellations and aircraft manufacturer or seating capacity.

[Place Table 5 about here]

We find a consistent and significant result across all estimated models: fewer cancellations for the last flight of day. This result highlights another distinction between flight delays and cancellations, since Mayer and Sinai (2003b) document more departure delays for last flight of day. To verify that the reduction in last flight of the day cancellations is not merely due to the fact that carriers typically have a surplus of idle aircraft late at night, we exam evening departures (8 p.m. to 9 p.m.) and night departures (9 p.m. to 10 p.m.). Last flight of day comprises $73 \%$ and $86 \%$ of the evening and night departure, respectively. We use separate scheduled departure intervals to control for the availability of idle/replacement aircraft and level of airport congestion. Carriers have fewer rescheduling options (i.e., alternative routings or other carriers' flights) when the last flight of day occurs later in the day. This may explain why the last flight of day for evening departures is neither more likely nor less likely to be canceled (see model 8). Whereas we find significantly fewer last flight of day cancellations for night departures (see model 9).

[Place Table 6 about here]

We also examined scheduled departures between 3 a.m. and 8 a.m. to see how carriers handled morning cancellations (i.e., they wake up with a broken plane). The results from model (10) reveal that many of the same factors (revenue, load factors, number of daily flights, poor weather, etc.) which influence cancellations throughout the day also generate early morning cancellations. We find a 1 percentage point reduction (40\% drop) in early morning cancellations for hub origination flights. On the other hand, early morning airline hub destination flights have significantly higher (21\% increase) cancellation rates. This is likely due to mandatory rest periods for flight crews (i.e., all flight crew members must have 9 consecutive hours of rest for each 24 hour period). Since most airline hub destination flights (88\%) are not originating from the carrier's hub, there are fewer substitute flight crews available to replace ineligible flight crews. 
Finally, we note that morning departures are more likely to cancel the last flight of day. We do not place too much importance to this result given that less than $5 \%$ of early morning departures receive the last flight of day designation and numerous rebooking alternatives exist for passengers displaced early in the morning.

The above results suggests that carriers limit passenger inconvenience and avoid costly overnight reimbursements by not canceling the last flight of day (especially at night) since this would force passengers to either rebook on a competing carrier or unexpectedly spend a night at the airport or departure city. Neither of these options is very appealing for the carrier, hence the lower cancellation rates for last flight of day. Alternatively, better performance for last flight of day could also be considered as evidence supporting the "flight network hypothesis" since getting the final daily flight to its scheduled destination sets the carrier up for regular operations the following day. In sum, we find considerable support for the third hypothesis that carriers minimize passenger inconvenience by not canceling fuller planes, flights on routes with infrequent scheduled service, and the last flight of day.

\subsubsection{Revenue Maximization}

The fourth and final hypothesized airline objective is that carriers maximize revenues. Average revenue is our preferred flight revenue proxy since it assumes that aircraft are carrying the average number of passengers for that route and carrier, whereas potential revenue represents an upper-bound of lost revenue from a flight cancellation due to the assumption that every seat is taken. The empirical estimations show overwhelming evidence (11 of 13 models) that average revenue is significant and inversely correlated with flight cancellations.

Table 7 reveals that average revenue has an impact at mid-size airports (100 to 400 total daily operations) and an even larger impact at small-size airports ( $<100$ total daily operations). The typical flight from a small airport involves a small plane, flying a short distance, with a high average fare, and is destined for a carrier's hub airport. Recall that average one-way fare is constructed from non-stop tickets, hence connecting passenger fares are excluded. Small airports also have the highest proportion of connecting passengers. ${ }^{23}$ Consequently, average revenue at small airports may serve as a rough proxy for actual flight 
revenue since it is compiled from a small subset of travelers who are purchasing non-stop tickets. Finally, discussions with a commuter airline operations manager reveal that a shortfall in flight revenue is sufficient justification for a flight cancellation. The manager also remarked that: "I wish our (mainline) parent company had the same policy".

[Place Table 7 about here]

We find no link between our alternative route revenue measure, potential revenue (see model 14) and flight cancellations. In addition, the yield results were also uninformative and hence not reported. In sum, we find some support for the hypothesis that carriers maximize revenues when making flight cancellation decisions. The revenue-flight cancellation link is most prominent at small and mid-size airports. Caution, however, should be exerted before inferring too much from these findings for three reasons. First, most of the average revenue results are sensitive to the empirical specification. ${ }^{24}$ Second, while we observe individual flight outcomes, we do not observe individual flight revenues. Instead, we use the lowest level of revenue data aggregation available to us, quarterly averages of route revenue. Given this crude revenue proxy, it is somewhat surprising that we are able to detect any pattern at all between revenues and flight cancellations. Third, we get different results across the three route revenue measures. While the airline industry typically uses yield to measure revenue, we opt to use a flight-based revenue proxy rather than a seat-based revenue variable. For example, a large aircraft (300+ seats) may have the same yield as a small regional jet (70 seats), yet the revenue from these flights will be substantially different. Improved flight-based revenue measures remain an area for future research.

[Place Table 8 about here]

\subsubsection{Logistical Issues}

We now briefly discuss other important logistical and weather variables. It is not surprising that airport congestion (measured by daily total airport operations) particularly at the destination is positively correlated with flight cancellations, since flight delays due to airport congestion have been well documented (Mayer and Sinai, 2003a; Mazzeo, 2003). This congestion result may explain why Southwest Airlines, the 
carrier with the best historical on-time performance record (Air Travel Consumer Report, March 2003), is reluctant to enter markets with congested airport facilities (Oh and Wiggins, 2001; Boguslaski et al. 2004). Model (11) reveals that most large U.S. airports (>400 daily take-offs and landings) are capacity constrained since an increase in scheduled flight operations is correlated with higher cancellation rates. The problem of airport congestion is most acute at the nation's four slot-controlled airports. Model (15) shows that both slot origination and destination airports have significantly higher cancellation rates. This result is likely due to the nature of a slot, which entitles the carrier to use the slot (land/depart typically within a 60 minute window) or lose it.

Time01 registers significantly higher cancellation rates in most estimated models which suggests an increase in flight cancellations for flights scheduled later in the day. A carrier may opt to cancel a flight rather than experience cascading delays (Mazzeo, 2003) for the remainder of the day. The airport size estimations in Table 7 indicate that the small airports experience the majority of late-in-the-day flight cancellations. For example, the marginal effect of 0.0132 for time01 in model (8) suggests that the cancellation rate for an 8 p.m. departure at small airports is 0.52 percentage points (or 20 percent) higher than an 8 a.m. departure.

Flight cancellation decisions appear to be independent of flight length since a majority of the flight cancellation models have insignificant estimates for short flight and middle distance. Not surprisingly, carriers that are experiencing labor unrest have higher cancellation rates. Finally, as expected, severe weather (rain and frozen precipitation) at both origination and destination airports increases the likelihood of a flight cancellation with frozen precipitation causing a larger service disruption than rain.

\subsection{Cancellations vs. Delays}

In this section we analyze the similarities and differences between the economics of flight cancellations and delays. ${ }^{25}$ In some instances cancellations and delays behave like substitute goods where a carrier can trade-off a flight cancellation to avoid additional delays. In other cases, cancellations and delays act like complementary goods that occur in unison and hence cannot be mitigated. First, we will present the evidence of a trade-off between cancellations and delays. We find that an increase in the number of 
additional planes needed to accommodate displaced passengers (i.e., higher load factor) reduces the flight cancellation rate, yet increases the likelihood of a flight delay (see model 7). Rupp et al. (2005) document a trade-off between cancellations and delays at slot-controlled airports during irregular operations as slot airports have more cancellations yet fewer delays. A flight cancellation and delay trade-off also occurs at hub airline airports with origination and destination hubs having fewer cancellations yet hub airlines are subject to more flight delays (Mayer and Sinai, 2003a).

Next, we also find some evidence that cancellations are merely extended flight delays, with the same economic and external factors that contribute to delays also causing flight cancellations. For example, we find worse service quality (more cancellations and departure delays) on less competitive routes. In addition, flight schedules appear to deteriorate during the day since time01 indicates that evening departures are subject to more cancellations and more departure delays than morning flights. We also find that airport congestion, labor unrest, and poor weather conditions all contribute to both more delays and cancellations.

Passengers may view flight cancellations and long delays differently since the rights to the airline seat are quite different. For example, after a flight cancellation all passengers lose their right to a seat on that flight, and hence must be re-accommodated. If there are not enough available seats on the next flight, some passengers will be forced to wait until an even later flight. In comparison during an extended flight delay, all passengers still retain their seat rights on the original flight.

\section{Conclusion}

This paper extends the literature on airline service quality by examining determinants of flight cancellations. Since the Airline Deregulation Act of 1978, airlines have attracted Congressional attention due to disgruntled passengers, employee strikes, proposed mergers, and financial concerns. This study proposes four possible airline flight operations objectives that carriers might pursue in order to satisfy existing flight schedules. We find that more competitive routes have lower cancellation rates. Since monopoly routes are especially prevalent at smaller "Podunk" airports which lack mechanics, we attribute the increase in flight cancellations on monopoly routes to an airport effect rather than a monopoly effect. We find considerable 
support for the hypothesis that carriers maintain their flight network by canceling flights to and from their hubs less frequently. Moreover, this hub airline effect (for both origination and destination airports) is strongest for large hub operations. There also is substantial evidence which shows that carriers minimize passenger inconvenience by not canceling fuller planes, infrequently served routes, and the final flight of the day. Finally, we find some support for the revenue maximization objective since the estimations reveal a significant reduction in cancellations on routes with higher average revenue.

There are some limitations for this research. Many factors that are observable to the airlines are not observable to us. For example, we treat airline's resources such as ground crews, flight crews, replacement aircraft, and maintenance personnel as exogenous. In fact, such decisions are endogenous for each airline. Carriers can have more reliable schedules if they build in more redundancy (i.e., stand-by flight crews and aircraft) in the schedule. Built-in redundancy, however, is a costly way to reduce flight cancellations. One way carriers manage their redundancy levels is by taking aircraft out-of-service for regularly scheduled maintenance checks on light travel days (i.e., Tuesdays and Wednesdays). This enables carriers to have more aircraft available and hence higher levels of redundancy on the busier travel days (i.e., holidays \& weekends). More redundancy on the weekends may explain the reduction in cancellations on Thursday, Fridays, and Sundays.

We are now able to address the public policy question posed in the introduction: how might airline consolidation influence flight cancellations? If consolidation causes a reduction in route competition (i.e., more monopolist routes), then these estimates suggest that an increase in flight cancellations would likely occur. Specifically, we estimate that a 10 percent increase in the number of monopoly routes in the U.S., holding everything else unchanged, would increase the cancellation rate by 0.023 percentage points (or about 1 percent).

More generally, what public policy implications can be gleaned from this analysis? Although severe weather events are clearly beyond the control of airlines, the findings that cancellation rates are significantly lower on Thursdays, Fridays and Sundays and on routes with infrequent daily service suggest that flight cancellations are not random events. Finally, given that we find considerable support for the hypothesis that carriers are limiting passenger inconvenience in addition to maintaining their existing flight networks, 
we see little need for active government intervention to improve service quality. Instead, we advocate for greater transparency from the U.S. Department of Transportation on the causes of flight cancellations. Specifically, the DOT should begin collecting data on the causes of flight cancellations (much like they have done for flight delays since June, 2003) and publicize their findings in order to break the misperception that all flight cancellations are beyond the carriers' control. This news might motivate carriers to improve service quality and may help weary air travelers sleep a little easier.

\section{References}

Air Transport Association. (2001). Annual Report 2001, Washington, D.C.

Boguslaski, C., Ito, H. and Lee, D. (2004). 'Entry Patterns in the Southwest Airlines Route System', Review of Industrial Organization, 25:3, pp. 317-350.

Borenstein, S. and Netz, J. (1999). 'Why Do All the Flights Leave at 8 am?: Competition and Departuretime Differentiation in Airline Markets', International Journal of Industrial Organization, 17, pp. $611-40$.

Brueckner, J.K. (2002). 'Airport Congestion when Carriers have Market Power', American Economic Review, 92:5, pp. 1357-1375.

Carey, S. (2000). 'Analysts Trim Profit Estimates for UAL Amid Worries About Flight Cancellations', Wall Street Journal, 14 August, A4.

Carey, S. and McCartney, S. (2001). 'Airline Workers Look to Congress for Aid - Employees Decry the Use of Force Majeure Clause, After Huge Bailout Plan', Wall Street Journal, 26 September, A3.

Dahl, J. (1991). 'Flight Cancellations Are a Rising Problem for Travelers', Wall Street Journal, 15 February, B1.

Davidson, R. and MacKinnon, J.G. (1993). Estimation and Inference in Econometrics. Oxford University Press: New York. 
Ellig, J. and Kelly, K. (2002). 'Competition and Quality in Deregulated Industries: Lessons for the Education Debate', Texas Review of Law 63 Politics, 6:2, pp. 335-397.

Foreman, S.E. and Shea, D.G. (1999). 'Publication of Information and Market Response: The Case of Airline on Time Performance Reports', Review of Industrial Organization, 14, pp. 147-62.

Härdle, W., Horowitz, J. and Kreiss, J. (2002). 'Bootstrap Methods for Time Series', working paper, CASE - Center for Applied Statistics and Economics, Humboldt-Universität zu Berlin, Germany.

Heller, J.E. (2000). 'Airline Campaigns to Improve Service Are Found Lacking', Wall Street Journal, 28 June, A1.

Hoxby, C. (2000). 'Does Competition Among Public Schools Benefit Students and Taxpayers?' American Economic Review, 90:5, 1209-38.

Kessler, D.P. and McClellan, M. (2000). 'Is Hospital Competition Socially Wasteful?', Quarterly Journal of Economics, 115:2, pp. 577-615.

Lee, D. and Ito, H. (2005). 'Assessing the Impact of September 11th Terrorist Attacks on U.S. Airline Demand', Journal of Economics and Business, 57:1, pp. 75-95.

Mayer, C. and Sinai, T. (2003a). 'Network Effects, Congestion Externalities, and Air Traffic Delays: or Why All Delays Are Not Evil', American Economic Review, 93:4, pp. 1194-1215.

Mayer, C. and Sinai, T. (2003b). 'Why Do Airline Schedules Systematically Underestimate Travel Time?', working paper, Wharton School of Business.

Mazzeo, M.J. (2003). 'Competition and Service Quality in the U.S. Airline Industry', Review of Industrial Organization, 22:4, pp. 275-296.

Morrison, S. and Winston, C. (1995). The Evolution of the Airline Industry. Washington, D.C.: The Brookings Institution.

(2000). Deregulation of Network Industries: What's Next? Edited by Sam Peltzman and Clifford Winston. Washington, D.C.: AEI-Brookings Joint Center for Regulatory Studies. 
Oh, J. and Wiggins, S.N. (2001). 'Fringe Entry, Price, and Quality: Southwest Airlines and Competition in the Airline Industry,' Texas A\&M University Department of Economics Working Paper.

Oum, T.H., Park, J. and Zhang, A. (2000). Globalization and Strategic Alliances: The Case of the Airline Industry. Amsterdam, Netherlands: Pergamon.

Rupp, N.G., Holmes, G.M. and DeSimone, J. (2005). 'Airline Schedule Recovery after Airport Closures: Empirical Evidence since September 11th", Southern Economic Journal, 71:4, pp. 800-20.

Rupp, N.G. (2005). 'Flight Delays and Cancellations', working paper, East Carolina University Department of Economics.

Suzuki, Y. (2000). 'The Relationship between On time Performance and Airline Market Share', Transportation Research: Part E: Logistics and Transportation Review, 36:2, pp. 139-54.

Thengvall, B.G., Bard, J.F. and Yu, G. (2000). 'Balancing User Preferences for Aircraft Schedule Recovery During Irregular Operations', IIE Transactions, 32, pp. 181-193.

Thenghall, B.G., Yu, G. and Bard, J.F. (2001). Multiple Fleet Aircraft Schedule Recovery Following Hub Closures', Transportation Research: Part A: Policy and Practice, 35:4, pp. 289-308.

US Department of Transportation. (2001). Office of Aviation Enforcement and Proceedings, Air Travel Consumer Report (January 2001, February 2001, and March 2003), Washington, D.C.: US Government Printing Office.

Vuong, Q.H. (1989). 'Likelihood Ratio Tests for Model Selection and Non-nested Hypotheses', Econometrica, 57:2, pp. 307-333.

Windle, R. and Dresner, M. (1999). 'Competitive Responses to Low Cost Carrier Entry', Transportation Research Part E, 35, pp. 59-75.

Yan, S. and Yang, D. (1996). 'A Decision Support Framework for Handling Schedule Perturbations', Transportation Research: Part B: Methodology, 30, pp. 405-419. 


\section{Notes}

${ }^{1}$ The complaint rate increase in the last couple of years is a relatively recent development since Morrison and Winston (2000, p. 20) show that complaints against U.S. carriers per billion revenue passenger miles are relatively constant between 1990 and 1998.

${ }^{2}$ We are aware of only two other studies examining cancellations. An earlier version of Mayer and Sinai (2003a) included one cancellation regression. This regression, however, was omitted in the final published version of the paper. Rupp et al. (2005) examine flight cancellations during irregular operations (i.e., security-related airport closures).

${ }^{3}$ http://www.transtats.bts.gov/OT_Delay/OT_DelayCause1.asp accessed 2 March 2004.

${ }^{4}$ For related flight delay literature see Foreman and Shea (1999).

${ }^{5}$ We also find shorter delays at more concentrated origination and destination airports.

${ }^{6}$ This variable is an approximation since we observe monthly load factor averages, rather than actual passenger bookings for specific flights. Further we assume every aircraft on the route has the same seating capacity.

${ }^{7} \mathrm{~A}$ few flights (primarily red-eye flights from the West to the East coast of the U.S.) are scheduled to depart the following day, shortly after midnight. Hence flights scheduled before 3:00 a.m. are treated as the "same day" for the last flight of day designation.

${ }^{8}$ While there is no FAA regulation that requires airlines to assist displaced passengers, most U.S. carriers provide overnight accommodations if the cancellation is caused by events within the carriers' control (e.g., see the Customer Service Plan for American Airlines at www.aa.com). On 17 February 2005, a new European Union (EU) rule came into effect that guarantees monetary compensation for airline passengers who are bumped or whose flights are delayed or canceled at all EU airports (europa.eu.int/comm/transport/air/rights/index_en.htm).

${ }^{9}$ Norman Strickland, Assistant Director for the Office of Aviation Enforcement and Proceedings, stated on 25 September 2001 that "refunds should be provided upon request to passengers who wish to cancel their trips as a result of a flight cancellation or significant schedule change made by the carrier" (airconsumer.ost.dot.gov/rules/20010925.htm).

${ }^{10}$ The Davidson-MacKinnon test is comparable to the $J$-test commonly used in testing regression models.

${ }^{11}$ Since the revenue measures are defined at the route level, we exclude indicator variables for route effects.

${ }^{12}$ The major carriers include Alaska, America West, American, Continental, Delta, Northwest, Southwest, TWA, United, and US Airways. In 2001, two additional major carriers were added: Aloha and American Eagle. These data exclude commuter airlines such as US Airways Express or Delta Connection. 
${ }^{13}$ See Lee and Ito (2005) for an analysis of how the September 11th terrorist attacks impacted U.S. airline demand.

${ }^{14}$ This is due to (i) missing tail numbers, (ii) incorrectly recorded tail numbers, and (iii) tail numbers that are no longer active in the FAA registry database. In situations where the tail number (and hence seating capacity) is unknown, seating capacity is found by substituting the median value of seats on comparable flights (i.e., same flight number, route, carrier, month, and year).

${ }^{15}$ In cases of missing weather data, we use the nearest weather reporting station within twenty-five miles.

${ }^{16}$ Since many NOAA weather stations do not report wind speed and daily snowfall totals, we must exclude wind as a weather variable. We construct our own snowfall measure by interacting temperature and precipitation.

${ }^{17}$ Two additional route competition measures were also considered: (i) the daily number of carriers serving route $r$; and (ii) effective competitors (the inverse of the Herfindahl index for all carriers serving route $r$ each day). Routes with more carriers have significantly fewer flight cancellations. We find, however, no link between the number of effective competitors and flight cancellations. These results are available upon request.

${ }^{18}$ Mayer and Sinai (2003b), however, use a sample of 3 million flights between 1988 and 2000 and find better on-time performance for monopoly routes.

${ }^{19}$ We also examined the competitive influences on service quality at the airport level by including airport concentration at both the origination and destination airports. We found little evidence that airport concentration affected flight cancellations in a predictable manner.

${ }^{20}$ For example, in May 2001, 83\% of all flights at small airports involve monopoly routes, whereas monopoly routes comprise $65 \%$ and $40 \%$ of flights from medium and large airports, respectively.

${ }^{21}$ These results are available upon request of the authors.

${ }^{22}$ We also find evidence that the linear seating capacity measure load factor is inversely correlated with flight cancellations. We opt to use the additional flights needed variable due to the large nonlinear effects of load factor.

${ }^{23}$ For example, in May 2001, the proportion of local passengers originating from small, medium, and large airports was $28.5 \%, 47.4 \%$, and $46.4 \%$ respectively.

${ }^{24}$ Specifically, average revenue loses its statistical significance in most estimations if the non-linear load factor (additional planes needed) is replaced by a linear load factor measure.

${ }^{25}$ For an in-depth look at the relationship between flight delays and cancellations see Rupp (2005) which uses a nested logit model to examine on-time flight arrivals, delays, and cancellations. 
Figure 1: Predicted vs. Actual Flight Cancellations

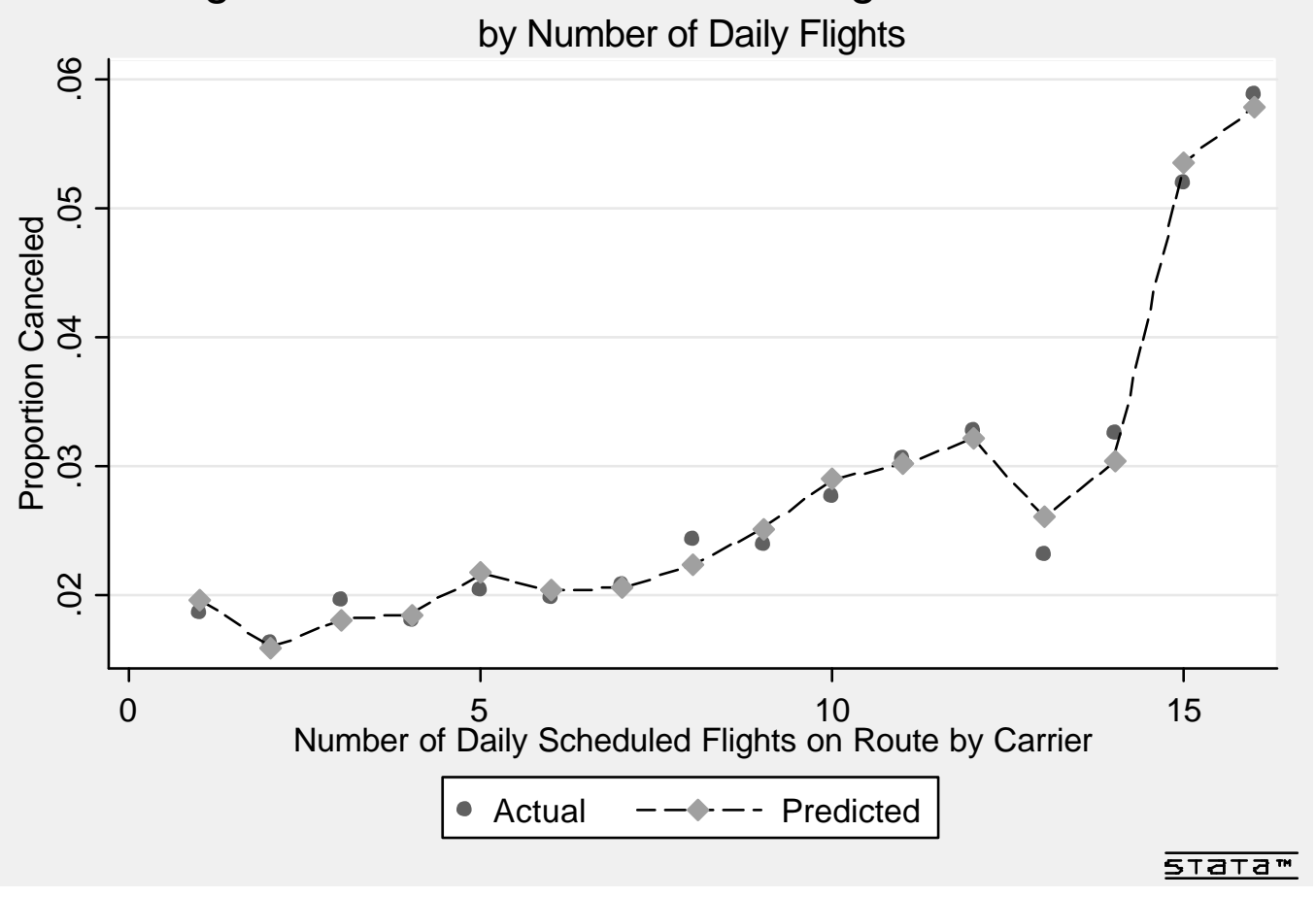


Table 1: Summary of Domestic Flights by U.S. Carriers On-time Performance ${ }^{1}, 1995-2002$

\begin{tabular}{|c|c|c|c|c|c|c|}
\hline Year & $\begin{array}{l}\text { Scheduled } \\
\text { Flights }\end{array}$ & $\begin{array}{c}\text { Flights } \\
\text { Canceled }\end{array}$ & $\begin{array}{l}\text { Pecent } \\
\text { On-time }\end{array}$ & $\begin{array}{l}\text { Percent } \\
\text { Late }\end{array}$ & $\begin{array}{l}\text { Percent } \\
\text { Canceled }\end{array}$ & $\begin{array}{l}\text { Percent } \\
\text { Diverted }\end{array}$ \\
\hline 1995 & $5,327,435$ & 91,905 & 76.65 & 21.43 & 1.73 & 0.20 \\
\hline 1996 & $5,351,983$ & 128,536 & 71.87 & 25.46 & 2.40 & 0.26 \\
\hline 1997 & $5,411,843$ & 97,763 & 75.91 & 22.06 & 1.81 & 0.22 \\
\hline 1998 & $5,384,721$ & 144,509 & 74.27 & 22.80 & 2.68 & 0.24 \\
\hline 1999 & $5,527,884$ & 154,311 & 73.07 & 23.89 & 2.79 & 0.25 \\
\hline 2000 & $5,683,047$ & 187,490 & 69.04 & 27.41 & 3.30 & 0.25 \\
\hline 2001 & $5,967,780$ & 231,198 & 73.07 & 23.89 & $2.79^{*}$ & 0.25 \\
\hline 2002 & $5,267,770$ & 64,981 & 80.75 & 17.86 & 1.23 & 0.16 \\
\hline Average & $5,490,308$ & 137,587 & 74.33 & 23.10 & 2.34 & 0.23 \\
\hline
\end{tabular}

${ }^{1}$ Source: Bureau of Transportation Statistics (www.bts.gov/oai/on_time_2002/), accessed 1/30/2004. U.S. carriers include: Alaska, America West, American, Continental, Delta, Northwest, Southwest, TWA, United, US Airways.

*Because of the shutdown of the air transportation system as a result of the terrorist attacks on September 11, 2001, the BTS granted air carriers waivers that we would not count the forced cancellations against the air carriers' on-time performance ratings. Hence, the "Percent Canceled" does not reflect these forced cancellations. For historical purposes, however, the number of "Flights canceled" includes all cancellations regardless of reason. 
Table 2: Descriptive Statistics: $5 \%$ sample of all daily domestic flights by U.S. carriers, January 1995 to August 2001

\begin{tabular}{|c|c|c|c|}
\hline Variable & Obs & Mean & Std. Dev. \\
\hline Proportion Canceled & $1,545,085$ & 0.0256 & 0.1578 \\
\hline Proportion Delayed & $1,545,085$ & 0.1857 & 0.3889 \\
\hline \multicolumn{4}{|l|}{ Economic Variables } \\
\hline Potential Revenue (in $\$ 10,000$ s) & $1,447,095$ & 0.3054 & 0.2145 \\
\hline Load Factor (Monthly Average) & $1,545,085$ & 0.6702 & 0.1253 \\
\hline Additional Planes Needed & $1,529,413$ & 2.6642 & 2.2392 \\
\hline Yield (dollars per revenue passenger mile) & $1,544,653$ & 0.3541 & 0.3283 \\
\hline Average One-way Fare (Quarterly Average) & $1,544,653$ & 165.9045 & 79.3885 \\
\hline Average Revenue (in $\$ 10,000$ s) & $1,447,096$ & 0.2073 & 0.1589 \\
\hline \multicolumn{4}{|l|}{ Route Competition Variables } \\
\hline Effective Competitors & $1,545,085$ & 1.4576 & 0.5833 \\
\hline Number of Carriers & $1,545,085$ & 1.6511 & 0.8351 \\
\hline Monopoly Route & $1,545,085$ & 0.5288 & 0.4992 \\
\hline Route Market Share & $1,545,085$ & 0.7809 & 0.2681 \\
\hline Small duopoly carrier & $1,545,085$ & 0.1012 & 0.3016 \\
\hline Large duopoly carrier & $1,545,085$ & 0.2036 & 0.4027 \\
\hline \multicolumn{4}{|l|}{ Airport Competition Variables } \\
\hline Airline Hub Origination & $1,545,085$ & 0.4052 & 0.4909 \\
\hline Large Airline Hub Origination & $1,545,085$ & 0.1680 & 0.3738 \\
\hline Medium Airline Hub Origination & $1,545,085$ & 0.1306 & 0.3370 \\
\hline Small Airline Hub Origination & $1,545,085$ & 0.1066 & 0.3087 \\
\hline Airline Hub Destination & $1,545,085$ & 0.4579 & 0.4982 \\
\hline Large Airline Hub Destination & $1,545,085$ & 0.1921 & 0.3940 \\
\hline Medium Airline Hub Destination & $1,545,085$ & 0.2056 & 0.4042 \\
\hline Small Airline Hub Destination & $1,545,085$ & 0.0601 & 0.2377 \\
\hline Airport Concentration Origination & $1,545,085$ & 0.0143 & 0.0822 \\
\hline Airport Concentration Destination & $1,545,085$ & 0.0156 & 0.0886 \\
\hline Slot Origination & $1,545,085$ & 0.1116 & 0.3148 \\
\hline Slot Destination & $1,545,085$ & 0.1659 & 0.3720 \\
\hline \multicolumn{4}{|l|}{ Aircraft Characteristics } \\
\hline Aircraft Age & $1,042,056$ & 11.1812 & 7.1492 \\
\hline Airbus & $1,123,649$ & 0.0443 & 0.2057 \\
\hline McDonnell-Douglas & $1,123,649$ & 0.2072 & 0.4053 \\
\hline Seating Capacity of Aircraft & $1,060,197$ & 162.2064 & 51.3258 \\
\hline \multicolumn{4}{|l|}{ Logistical Variables } \\
\hline Daily Total Airport Operations Orig. & $1,545,085$ & 6.1891 & 4.4511 \\
\hline Daily Total Airport Operations Dest & $1,545,085$ & 6.7667 & 5.0148 \\
\hline Daily Scheduled Flights & $1,545,085$ & 7.0840 & 4.7813 \\
\hline Last Flight of Day & $1,545,085$ & 0.1776 & 0.3822 \\
\hline Time01 & $1,545,085$ & 0.5660 & 0.1974 \\
\hline Short Flight (< 400 miles $)$ & $1,545,085$ & 0.4419 & 0.4966 \\
\hline Middle Distance (400 to 800 miles) & $1,545,085$ & 0.3429 & 0.4747 \\
\hline Labor Unrest & $1,545,085$ & 0.0585 & 0.2346 \\
\hline \multicolumn{4}{|l|}{ Weather Variables } \\
\hline Minimum Temperature Origination & $1,545,085$ & 51.1134 & 17.1647 \\
\hline Minimum Temperature Destination & $1,545,085$ & 49.7770 & 18.3997 \\
\hline Rain Origination & $1,545,085$ & 9.6895 & 31.6288 \\
\hline Rain Destination & $1,545,085$ & 9.8150 & 32.3273 \\
\hline Frozen Precipitation Origination & $1,545,085$ & 0.6169 & 5.0238 \\
\hline Frozen Precipitation Destination & $1,545,085$ & 0.7420 & 5.5027 \\
\hline
\end{tabular}


Table 3: Probit Estimations of Flight Cancellations - The Effect of Route Competition

Dependent Variable: Flight Cancellations for Domestic Flights by U.S. Carriers, January 1995 - August 2001.

\begin{tabular}{|c|c|c|c|c|c|c|c|c|c|}
\hline \multirow[t]{2}{*}{ Model } & \multicolumn{3}{|c|}{$(1)$} & \multicolumn{3}{|c|}{$(2)$} & \multicolumn{3}{|c|}{ (3) } \\
\hline & Coeff & Std. Error & Marg. Eff. & Coeff & Std. Error & Marg. Eff. & Coeff & Std. Error & Marg. Eff. \\
\hline \multicolumn{10}{|l|}{ Route Competition Variables } \\
\hline Monopoly & $0.0527 * *$ & 0.0103 & 0.0023 & & & & $0.0630 * *$ & 0.0149 & 0.0028 \\
\hline Market Share & & & & 0.0385 & 0.0203 & 0.0017 & & & \\
\hline Large Duopoly Carrier & & & & & & & 0.0065 & 0.0140 & 0.0003 \\
\hline Small Duopoly Carrier & & & & & & & $0.0370 *$ & 0.0178 & 0.0017 \\
\hline \multicolumn{10}{|l|}{ Hub Variables } \\
\hline Airline Hub Origination & -0.1672 ** & 0.0139 & -0.0071 & -0.1656 ** & 0.0141 & -0.0071 & -0.1652 ** & 0.0143 & -0.0070 \\
\hline Airline Hub Destination & $-0.1544^{* *}$ & 0.0136 & -0.0067 & $-0.1427^{* *}$ & 0.0133 & -0.0062 & -0.1580 ** & 0.0138 & -0.0069 \\
\hline \multicolumn{10}{|l|}{ Economic Variables } \\
\hline Average Revenue (in $\$ 10,000$ s) & $-0.0999 *$ & 0.0470 & -0.0044 & $-0.0970 *$ & 0.0465 & -0.0043 & -0.0950 * & 0.0473 & -0.0042 \\
\hline Additional Planes Needed & $-0.0957^{* *}$ & 0.0045 & -0.0042 & $-0.0943 * *$ & 0.0045 & -0.0042 & $-0.0951 * *$ & 0.0045 & -0.0042 \\
\hline \multicolumn{10}{|l|}{ Logistical Variables } \\
\hline Daily Total Airport Operations Orig. & 0.0012 & 0.0014 & 0.0001 & 0.0002 & 0.0014 & 0.0000 & 0.0007 & 0.0014 & 0.0000 \\
\hline Daily Total Airport Operations Dest & $0.0074 * *$ & 0.0014 & 0.0003 & 0.0058 ** & 0.0015 & 0.0003 & $0.0072 * *$ & 0.0014 & 0.0003 \\
\hline Daily Scheduled Flights & 0.0348 ** & 0.0011 & 0.0015 & $0.0352 * *$ & 0.0012 & 0.0015 & $0.0352 * *$ & 0.0011 & 0.0015 \\
\hline Last Flight of Day & $-0.0594 * *$ & 0.0094 & -0.0025 & $-0.0524 * *$ & 0.0095 & -0.0022 & $-0.0580 * *$ & 0.0093 & -0.0025 \\
\hline Time01 & 0.0361 * & 0.0177 & 0.0016 & 0.0281 & 0.0177 & 0.0012 & 0.0356 * & 0.0176 & 0.0016 \\
\hline Short Flight (< 400 miles $)$ & -0.0104 & 0.0154 & -0.0005 & -0.0030 & 0.0154 & -0.0001 & -0.0112 & 0.0154 & -0.0005 \\
\hline Middle Distance (400 to 800 miles) & -0.0161 & 0.0154 & -0.0007 & -0.0156 & 0.0154 & -0.0007 & -0.0173 & 0.0156 & -0.0008 \\
\hline Labor Unrest & 0.3098 ** & 0.0190 & 0.0182 & $0.3103^{* *}$ & 0.0191 & 0.0183 & 0.3098 ** & 0.0190 & 0.0182 \\
\hline \multicolumn{10}{|l|}{ Weather Variables } \\
\hline Minimum Temperature Origination & 0.0000 & 0.0004 & 0.0000 & -0.0002 & 0.0004 & 0.0000 & -0.0001 & 0.0004 & 0.0000 \\
\hline Minimum Temperature Destination & $-0.0033^{* *}$ & 0.0005 & -0.0001 & $-0.0033^{* *}$ & 0.0005 & -0.0001 & $-0.0032 * *$ & 0.0005 & -0.0001 \\
\hline Rain Origination & 0.0022 ** & 0.0001 & 0.0001 & $0.0022 * *$ & 0.0001 & 0.0001 & 0.0022 ** & 0.0001 & 0.0001 \\
\hline Rain Destination & $0.0022 * *$ & 0.0001 & 0.0001 & $0.0022 * *$ & 0.0001 & 0.0001 & $0.0022 * *$ & 0.0001 & 0.0001 \\
\hline Frozen Precipitation Origination & $0.0122 * *$ & 0.0005 & 0.0005 & $0.0122 * *$ & 0.0005 & 0.0005 & $0.0122 * *$ & 0.0005 & 0.0005 \\
\hline Frozen Precipitation Destination & 0.0106 ** & 0.0005 & 0.0005 & $0.0106^{* *}$ & 0.0005 & 0.0005 & $0.0106^{* *}$ & 0.0005 & 0.0005 \\
\hline Constant & $-1.7813^{* *}$ & 0.0475 & & $-1.7728^{* *}$ & 0.0483 & & $-1.7950 * *$ & 0.0469 & \\
\hline Log Likelihood & & $-155,175$ & & & $-155,204$ & & & $-155,169$ & \\
\hline Pseudo R-squared & & 0.086 & & & 0.086 & & & 0.086 & \\
\hline Number of Observations & & $1,447,096$ & & & $1,447,096$ & & & $1,447,096$ & \\
\hline
\end{tabular}

Note: Bootstrapped standard errors are reported. Regressions include carrier, day of week, month, and year dummy variables.

Marginal effects are defined as the effect on the probability that the average flight is cancelled. * and ${ }^{* *}$ indicate significance at the $5 \%$ and $1 \%$ levels. 
Table 4: Flight Cancellation Probits - The Effect of Airline Hubbing

Dependent Variable: Flight Cancellations for Domestic Flights by U.S. Carriers, January 1995 - August 2001.

\begin{tabular}{|c|c|c|c|c|c|c|c|}
\hline \multirow[t]{2}{*}{ Model } & \multicolumn{3}{|c|}{ (4) } & \multicolumn{4}{|c|}{ (5) } \\
\hline & Coeff & Std. Error & Marg. Eff. & Coeff & & Std. Error & Marg. Eff. \\
\hline \multicolumn{8}{|l|}{ Route Competition Variable } \\
\hline Monopoly & 0.0969 ** & 0.0124 & 0.0042 & 0.0310 & & 0.0305 & 0.0013 \\
\hline \multicolumn{8}{|l|}{ Hub Size Variables } \\
\hline Large Airline Hub Origination & -0.2910 ** & 0.0230 & -0.0105 & -0.2469 & ** & 0.0394 & -0.0087 \\
\hline Medium Airline Hub Origination & -0.2660 ** & 0.0192 & -0.0095 & -0.1342 & ** & 0.0384 & -0.0050 \\
\hline Small Airline Hub Origination & $-0.0982 * *$ & 0.0147 & -0.0040 & -0.0572 & * & 0.0250 & -0.0023 \\
\hline Large Airline Hub Destination & $-0.2766^{* *}$ & 0.0227 & -0.0102 & -0.1482 & ** & 0.0430 & -0.0056 \\
\hline Medium Airline Hub Destination & $-0.2188^{* *}$ & 0.0170 & -0.0084 & -0.1172 & ** & 0.0378 & -0.0045 \\
\hline Small Airline Hub Destination & -0.1846 ** & 0.0230 & -0.0068 & -0.0734 & * & 0.0361 & -0.0028 \\
\hline \multicolumn{8}{|l|}{ Economic Variables } \\
\hline Average Revenue (in $\$ 10,000$ s) & -0.1556 ** & 0.0451 & -0.0068 & -0.5146 & ** & 0.0559 & -0.0213 \\
\hline Additional Planes Needed & $-0.0953^{* *}$ & 0.0045 & -0.0042 & -0.0568 & ** & 0.0047 & -0.0024 \\
\hline \multicolumn{8}{|l|}{ Logistical Variables } \\
\hline Daily Total Airport Operations Orig. & $0.0076^{* *}$ & 0.0016 & 0.0003 & 0.0065 & & 0.0079 & 0.0003 \\
\hline Daily Total Airport Operations Dest & 0.0132 ** & 0.0016 & 0.0006 & 0.0323 & ** & 0.0063 & 0.0013 \\
\hline Daily Scheduled Flights & $0.0351 * *$ & 0.0011 & 0.0015 & 0.0258 & ** & 0.0023 & 0.0011 \\
\hline Last Flight of Day & $-0.0612^{* *}$ & 0.0094 & -0.0026 & -0.0718 & ** & 0.0100 & -0.0028 \\
\hline Time01 & $0.0410 *$ & 0.0177 & 0.0018 & 0.0571 & ** & 0.0184 & 0.0024 \\
\hline Short Flight $(<400$ miles $)$ & 0.0041 & 0.0155 & 0.0002 & -0.0734 & & 0.0375 & -0.0030 \\
\hline Middle Distance (400 to 800 miles) & -0.0038 & 0.0154 & -0.0002 & -0.1040 & & 0.0621 & -0.0042 \\
\hline Labor Unrest & $0.3081^{* *}$ & 0.0189 & 0.0180 & 0.3258 & ** & 0.0184 & 0.0184 \\
\hline \multicolumn{8}{|l|}{ Weather Variables } \\
\hline Minimum Temperature Origination & -0.0006 & 0.0004 & 0.0000 & 0.0005 & & 0.0005 & 0.0000 \\
\hline Minimum Temperature Destination & $-0.0030 * *$ & 0.0004 & -0.0001 & -0.0026 & ** & 0.0005 & -0.0001 \\
\hline Rain Origination & 0.0022 ** & 0.0001 & 0.0001 & 0.0022 & ** & 0.0001 & 0.0001 \\
\hline Rain Destination & $0.0021^{* *}$ & 0.0001 & 0.0001 & 0.0022 & ** & 0.0001 & 0.0001 \\
\hline Frozen Precipitation Origination & 0.0122 ** & 0.0005 & 0.0005 & 0.0123 & ** & 0.0005 & 0.0005 \\
\hline Frozen Precipitation Destination & 0.0106 ** & 0.0005 & 0.0005 & 0.0104 & ** & 0.0005 & 0.0004 \\
\hline Constant & $-1.8142^{* *}$ & 0.0481 & & -1.7049 & & $--^{\top}$ & \\
\hline Airport Fixed Effects & & No & & & & Yes & \\
\hline Log Likelihood & & $-155,044$ & & & & $-153,205$ & \\
\hline Pseudo R-squared & & 0.087 & & & & 0.0971 & \\
\hline Number of Observations & & $1,447,096$ & & & & $1,444,774$ & \\
\hline
\end{tabular}

Note: Bootstrapped standard errors are reported. Regressions include carrier, day of week, month, and year dummy variables. Marginal effects are defined as the effect on the probability that the average flight is cancelled. ${ }^{*}$ and ${ }^{* *}$ indicate $5 \%$ and $1 \%$ significance levels, respectively.

${ }^{1}$ Bootstrapped standard error for the constant is not consistent, since the regressions are not guaranteed to have the identical excluded referent airports across the bootstrapped samples. 
Table 5: Probit Estimations of Flight Cancellations \& Delays - The Effect of Aircraft Characteristics Flight Cancellations and Departure Delays ${ }^{1}$ for Domestic Flights by U.S. Carriers, January 1995 - August 2001.

\begin{tabular}{|c|c|c|c|c|c|c|}
\hline \multirow[t]{2}{*}{$\begin{array}{l}\text { Dependent Variable } \\
\text { Model }\end{array}$} & \multicolumn{3}{|c|}{$\begin{array}{l}\text { Flight Cancellation } \\
\text { (6) }\end{array}$} & \multicolumn{3}{|c|}{$\begin{array}{l}\text { Departure Delay } \\
\text { (7) }\end{array}$} \\
\hline & Coeff & Std. Error & Marg. Eff. & Coeff & Std. Error & Marg. Eff. \\
\hline \multicolumn{7}{|l|}{ Route Competition Variable } \\
\hline Monopoly & $0.0970 * *$ & 0.0209 & 0.0000 & $0.0569 * *$ & 0.0066 & 0.0145 \\
\hline \multicolumn{7}{|l|}{ Hub Size Variables } \\
\hline Airline Hub Origination & $-0.1317^{* *}$ & 0.0248 & 0.0000 & 0.0062 & 0.0088 & 0.0016 \\
\hline Airline Hub Destination & $-0.1055 * *$ & 0.0241 & 0.0000 & $-0.1003 * *$ & 0.0093 & -0.0256 \\
\hline \multicolumn{7}{|l|}{ Economic Variables } \\
\hline Average Revenue (in $\$ 10,000$ s) & $-0.2930 *$ & 0.1349 & -0.0001 & $0.1353^{* *}$ & 0.0204 & 0.0346 \\
\hline Additional Planes Needed & $-0.0643 * *$ & 0.0092 & 0.0000 & $0.0114 * *$ & 0.0012 & 0.0029 \\
\hline \multicolumn{7}{|l|}{ Aircraft Characteristics } \\
\hline Aircraft Age & $0.0048^{* *}$ & 0.0010 & 0.0000 & & & \\
\hline Airbus & 0.0413 & 0.0305 & 0.0000 & & & \\
\hline McDonnell-Douglas & 0.0012 & 0.0182 & 0.0000 & & & \\
\hline Seating capacity of aircraft & -0.0003 & 0.0002 & 0.0000 & & & \\
\hline \multicolumn{7}{|l|}{ Logistical Variables } \\
\hline Daily Total Airport Operations Orig. & $0.0075 * *$ & 0.0026 & 0.0000 & $0.0123 * *$ & 0.0008 & 0.0031 \\
\hline Daily Total Airport Operations Dest & $0.0089 * *$ & 0.0030 & 0.0000 & $0.0108 * *$ & 0.0009 & 0.0028 \\
\hline Daily Scheduled Flights & 0.0264 ** & 0.0033 & 0.0000 & 0.0058 ** & 0.0009 & 0.0015 \\
\hline Last Flight of Day & -0.0262 & 0.0171 & 0.0000 & $-0.0805^{* *}$ & 0.0059 & -0.0201 \\
\hline Time01 & -0.0006 & 0.0366 & 0.0000 & 1.1965 ** & 0.0126 & 0.3063 \\
\hline Short Flight (< 400 miles) & $-0.0871 * *$ & 0.0281 & 0.0000 & -0.0157 & 0.0112 & -0.0040 \\
\hline Middle Distance (400 to 800 miles) & -0.0916 ** & 0.0289 & 0.0000 & $0.0499 * *$ & 0.0097 & 0.0129 \\
\hline Labor Unrest & $0.1933^{* *}$ & 0.0303 & 0.0001 & 0.2428 ** & 0.0129 & 0.0683 \\
\hline \multicolumn{7}{|l|}{ Weather Variables } \\
\hline Minimum Temperature Origination & $-0.0040 * *$ & 0.0006 & 0.0000 & $-0.0007^{* *}$ & 0.0003 & -0.0002 \\
\hline Minimum Temperature Destination & -0.0011 & 0.0007 & 0.0000 & $-0.0012 * *$ & 0.0003 & -0.0003 \\
\hline Rain Origination & $0.0023^{* *}$ & 0.0002 & 0.0000 & $0.0036^{* *}$ & 0.0001 & 0.0009 \\
\hline Rain Destination & $0.0018 * *$ & 0.0002 & 0.0000 & $0.0025 * *$ & 0.0001 & 0.0006 \\
\hline Frozen Precipitation Origination & 0.0132 ** & 0.0009 & 0.0000 & $0.0111^{* *}$ & 0.0004 & 0.0028 \\
\hline Frozen Precipitation Destination & 0.0100 ** & 0.0008 & 0.0000 & 0.0078 ** & 0.0004 & 0.0020 \\
\hline Constant & $-8.2865 * *$ & 0.3470 & & $-1.9450 * *$ & 0.0287 & \\
\hline Log Likelihood & & $-29,215$ & & & $-654,924$ & \\
\hline Pseudo R-squared & & 0.215 & & & 0.060 & \\
\hline Number of Observations & & 749,467 & & & $1,447,096$ & \\
\hline
\end{tabular}

Note: Bootstrapped standard errors are reported. Regressions include carrier, day of week, month, and year dummy variables. Marginal effects are defined as the effect on the probability that the average flight is cancelled. ${ }^{*}$ and ${ }^{* *}$ indicate $5 \%$ and $1 \%$ significance levels, respectively. 
Table 6: Probit Estimations of Flight Cancellations - The Effect of Scheduled Departure Time

Dependent Variable: Flight Cancellations for Domestic Flights by U.S. Carriers, January 1995 - August 2001.

\begin{tabular}{|c|c|c|c|c|c|c|c|c|c|}
\hline \multirow{2}{*}{$\begin{array}{l}\text { Model } \\
\text { Scheduled Departure Time: }\end{array}$} & \multicolumn{3}{|c|}{$\begin{array}{c}(8) \\
\text { Evening }(8 p m-9 p m)\end{array}$} & \multicolumn{3}{|c|}{$\begin{array}{c}(9) \\
\text { Night (9pm - 10pm) }\end{array}$} & \multicolumn{3}{|c|}{$\begin{array}{c}\text { (10) } \\
\text { Early Morning (3am - 8am) }\end{array}$} \\
\hline & Coeff & Std. Error & Marg. Eff. & Coeff & Std. Error & $\begin{array}{l}\text { pm) } \\
\text { Marg. Eff. }\end{array}$ & Coeff & Std. Error & Marg. Eff. \\
\hline \multicolumn{10}{|l|}{ Route Competition Variables } \\
\hline Monopoly & 0.2930 ** & 0.0731 & 0.0091 & $0.5507^{* *}$ & 0.1600 & 0.0150 & 0.0582 & 0.0363 & 0.0024 \\
\hline \multicolumn{10}{|l|}{ Hub Variables } \\
\hline Airline Hub Origination & $-0.2624 * *$ & 0.0701 & -0.0082 & -0.4784 & 0.1436 & -0.0137 & $-0.2463^{\star *}$ & 0.0659 & -0.0103 \\
\hline Airline Hub Destination & -0.0233 & 0.0912 & -0.0007 & 0.0032 & 0.1284 & 0.0001 & 0.1249 * & 0.0551 & 0.0054 \\
\hline \multicolumn{10}{|l|}{ Economic Variables } \\
\hline Average Revenue (in $\$ 10,000$ s) & $-0.8542 *$ & 0.3678 & -0.0266 & -0.1194 & 0.2638 & -0.0031 & $-1.1597 * *$ & 0.2503 & -0.0470 \\
\hline \multirow{2}{*}{\multicolumn{10}{|c|}{ Logistical Variables }} \\
\hline & & & & & & & & & \\
\hline Daily Total Airport Operations Orig. & -0.0103 & 0.0067 & -0.0003 & 0.0203 & 0.0152 & 0.0005 & 0.0078 & 0.0075 & 0.0003 \\
\hline Daily Total Airport Operations Dest & -0.0098 & 0.0107 & -0.0003 & 0.0087 & 0.0213 & 0.0002 & 0.0048 & 0.0051 & 0.0002 \\
\hline Daily Scheduled Flights & $0.0172 * *$ & 0.0065 & 0.0005 & -0.0098 & 0.0129 & -0.0003 & $0.0457^{* *}$ & 0.0040 & 0.0019 \\
\hline Last Flight of Day & 0.0155 & 0.0594 & 0.0005 & -0.3039 ** & 0.0793 & -0.0095 & $0.1484^{* *}$ & 0.0426 & 0.0058 \\
\hline Time01 & 2.2234 & 1.8516 & 0.0693 & $-8.0084 * *$ & 2.4284 & -0.2054 & $-3.8509 * *$ & 1.2472 & -0.1560 \\
\hline Short Flight (< 400 miles) & $0.4219 * *$ & 0.1154 & 0.0127 & 0.2320 & 0.1727 & 0.0062 & 0.0394 & 0.0841 & 0.0016 \\
\hline Middle Distance (400 to 800 miles) & $0.7830 * *$ & 0.1306 & 0.0409 & $0.6346^{* *}$ & 0.2173 & 0.0316 & 0.0049 & 0.0934 & 0.0002 \\
\hline Labor Unrest & $0.2377^{* *}$ & 0.0765 & 0.0095 & 0.4792 ** & 0.0741 & 0.0202 & $0.4343 * *$ & 0.0603 & 0.0269 \\
\hline \multicolumn{10}{|l|}{ Weather Variables } \\
\hline Minimum Temperature Origination & 0.0016 & 0.0018 & 0.0000 & 0.0007 & 0.0024 & 0.0000 & 0.0018 & 0.0015 & 0.0001 \\
\hline Minimum Temperature Destination & -0.0036 & 0.0020 & -0.0001 & -0.0023 & 0.0022 & -0.0001 & 0.0005 & 0.0016 & 0.0000 \\
\hline Rain Origination & $0.0033 * *$ & 0.0004 & 0.0001 & $0.0035 * *$ & 0.0004 & 0.0001 & $0.0038^{* *}$ & 0.0002 & 0.0002 \\
\hline Rain Destination & 0.0021 ** & 0.0004 & 0.0001 & $0.0028^{* *}$ & 0.0005 & 0.0001 & 0.0030 ** & 0.0003 & 0.0001 \\
\hline Frozen Precipitation Origination & 0.0118 ** & 0.0017 & 0.0004 & 0.0136 ** & 0.0025 & 0.0003 & $0.0163^{* *}$ & 0.0015 & 0.0007 \\
\hline Frozen Precipitation Destination & 0.0110 ** & 0.0017 & 0.0003 & $0.0073^{* *}$ & 0.0020 & 0.0002 & 0.0102 ** & 0.0013 & 0.0004 \\
\hline Constant & $-4.4770^{* *}$ & 1.6788 & & $5.1172 *$ & 2.3890 & & 0.8731 & 1.0531 & \\
\hline Log Likelihood & & $-3,634$ & & & $-2,577$ & & & $-7,054$ & \\
\hline Pseudo R-squared & & 0.116 & & & 0.159 & & & 0.157 & \\
\hline Number of Observations & & 42,270 & & & 31,036 & & & 62,807 & \\
\hline Last Flight of Day (Percentage) & & $73.0 \%$ & & & $85.8 \%$ & & & $4.9 \%$ & \\
\hline
\end{tabular}

Note: Bootstrapped standard errors are reported. Regressions include carrier, day of week, month, and year dummy variables.

Marginal effects are defined as the effect on the probability that the average flight is cancelled. * and ${ }^{*}$ indicate significance at the $5 \%$ and $1 \%$ levels. 
Table 7: Probit Estimations of Flight Cancellations - The Effect of Airport Size

Dependent Variable: Flight Cancellations for Domestic Flights by U.S. Carriers, January 1995 - August 2001.

\begin{tabular}{|c|c|c|c|c|c|c|c|c|c|}
\hline \multirow{2}{*}{$\begin{array}{l}\text { Model } \\
\text { Sample }\end{array}$} & \multirow{2}{*}{\multicolumn{3}{|c|}{$\begin{array}{c}\text { (11) } \\
\text { Large Airports }\end{array}$}} & \multirow{2}{*}{\multicolumn{3}{|c|}{$\begin{array}{c}(12) \\
\text { Medium Airports }\end{array}$}} & \multirow{2}{*}{\multicolumn{3}{|c|}{$\begin{array}{c}(13) \\
\text { Small Airports }\end{array}$}} \\
\hline & & & & & & & & & \\
\hline Route Competition Variables & & & & & & & & & \\
\hline $\begin{array}{l}\text { Monopoly } \\
\text { Hub Variables }\end{array}$ & 0.0896 ** & 0.0135 & 0.0043 & $-0.0958 *$ & 0.0207 & -0.0033 & 0.0798 & 0.0740 & 0.0038 \\
\hline Airline Hub Origination & $-0.1923 * *$ & 0.0189 & -0.0092 & $-0.1623 *$ & 0.0288 & -0.0047 & & & \\
\hline $\begin{array}{l}\text { Airline Hub Destination } \\
\text { Economic Variables }\end{array}$ & $-0.1605^{* *}$ & 0.0177 & -0.0072 & $-0.1290 *$ & 0.0300 & -0.0042 & -0.0505 & 0.0595 & -0.0026 \\
\hline Average Revenue (in $\$ 10,000$ s) & -0.0429 & 0.0517 & -0.0020 & -0.5110 * & 0.0965 & -0.0166 & -1.0304 ** & 0.2468 & -0.0518 \\
\hline $\begin{array}{l}\text { Additional Planes Needed } \\
\text { Logistical Variables }\end{array}$ & $-0.1105^{* *}$ & 0.0052 & -0.0052 & -0.0598 * & 0.0095 & -0.0019 & $-0.0600 * *$ & 0.0112 & -0.0030 \\
\hline Daily Total Airport Operations Orig. & 0.0070 ** & 0.0018 & 0.0003 & -0.0139 & 0.0124 & -0.0005 & 0.0161 & 0.0529 & 0.0008 \\
\hline Daily Total Airport Operations Dest & 0.0101 ** & 0.0018 & 0.0005 & 0.0002 & 0.0038 & 0.0000 & -0.0064 & 0.0041 & -0.0003 \\
\hline Daily Scheduled Flights & 0.0370 ** & 0.0013 & 0.0017 & 0.0487 * & 0.0028 & 0.0016 & $0.0264^{* *}$ & 0.0065 & 0.0013 \\
\hline Last Flight of Day & $-0.0878 * *$ & 0.0112 & -0.0038 & -0.0752 * & 0.0195 & -0.0023 & -0.0466 & 0.0239 & -0.0023 \\
\hline Time01 & 0.0394 & 0.0205 & 0.0018 & 0.0244 & 0.0413 & 0.0008 & 0.2626 ** & 0.0620 & 0.0132 \\
\hline Short Flight (< 400 miles) & $-0.0743 * *$ & 0.0197 & -0.0034 & -0.1876 * & 0.0400 & -0.0060 & 0.1907 * & 0.0947 & 0.0085 \\
\hline Middle Distance (400 to 800 miles) & $-0.0726 * *$ & 0.0193 & -0.0033 & $-0.1087 *$ & 0.0336 & -0.0034 & 0.0094 & 0.0914 & 0.0005 \\
\hline $\begin{array}{l}\text { Labor Unrest } \\
\text { Weather Variables }\end{array}$ & $0.2954^{* *}$ & 0.0210 & 0.0181 & 0.4074 * & 0.0398 & 0.0202 & $0.2413^{* *}$ & 0.0418 & 0.0152 \\
\hline Minimum Temperature Origination & -0.0002 & 0.0005 & 0.0000 & 0.0005 & 0.0008 & 0.0000 & $0.0028 *$ & 0.0010 & 0.0001 \\
\hline Minimum Temperature Destination & $-0.0037^{* *}$ & 0.0005 & -0.0002 & $-0.0018 *$ & 0.0008 & -0.0001 & -0.0064 * & 0.0012 & -0.0003 \\
\hline Rain Origination & $0.0026^{* *}$ & 0.0001 & 0.0001 & 0.0014 * & 0.0002 & 0.0000 & 0.0020 ** & 0.0002 & 0.0001 \\
\hline Rain Destination & 0.0022 ** & 0.0001 & 0.0001 & $0.0021 *$ & 0.0002 & 0.0001 & $0.0021 * *$ & 0.0001 & 0.0001 \\
\hline Frozen Precipitation Origination & $0.0130 * *$ & 0.0007 & 0.0006 & $0.0109 *$ & 0.0011 & 0.0004 & $0.0109 * *$ & 0.0009 & 0.0005 \\
\hline Frozen Precipitation Destination & $0.0107^{* *}$ & 0.0007 & 0.0005 & $0.0109 *$ & 0.0010 & 0.0004 & $0.0094 * *$ & 0.0011 & 0.0005 \\
\hline Constant & $-1.7722 * *$ & 0.0625 & & $-1.7928 *$ & 0.1060 & & -1.9378 * & 0.2014 & \\
\hline Log Likelihood & & $-106,497$ & & & $-29,122$ & & & $-18,898$ & \\
\hline Pseudo R-squared & & 0.093 & & & 0.075 & & & 0.064 & \\
\hline Number of Observations & & 926,628 & & & 357,360 & & & 163,108 & \\
\hline
\end{tabular}


Table 8: Flight Cancellation Probits - The Effect of Flight Revenue \& Slot Controlled Airports Dependent Variable: Flight Cancellations for Domestic Flights by U.S. Carriers, January 1995 - August 2001.

\begin{tabular}{|c|c|c|c|c|c|c|}
\hline \multirow[t]{2}{*}{ Model } & \multicolumn{3}{|c|}{ (14) } & \multicolumn{3}{|c|}{ (15) } \\
\hline & Coeff & Std. Error & Marg. Eff. & Coeff & Std. Error & Marg. Eff. \\
\hline \multicolumn{7}{|l|}{ Route Competition Variable } \\
\hline Monopoly & $0.0523^{* *}$ & 0.0103 & 0.0023 & $0.0702 * \star$ & 0.0106 & 0.0031 \\
\hline \multicolumn{7}{|l|}{ Hub Variables } \\
\hline Airline Hub Origination & $-0.1745^{* *}$ & 0.0139 & -0.0074 & $-0.1193 * *$ & 0.0139 & -0.0051 \\
\hline Airline Hub Destination & $-0.1596 * *$ & 0.0136 & -0.0069 & $-0.1034 * \star$ & 0.0137 & -0.0045 \\
\hline Slot Origination & & & & $0.1327^{* *}$ & 0.0160 & 0.0065 \\
\hline Slot Destination & & & & $0.1765 * *$ & 0.0149 & 0.0088 \\
\hline \multicolumn{7}{|l|}{ Economic Variables } \\
\hline Potential Revenue (in $\$ 10,000$ s) & -0.0045 & 0.0298 & -0.0002 & & & \\
\hline Average Revenue (in $\$ 10,000$ s) & & & & $-0.2014^{* *}$ & 0.0469 & -0.0088 \\
\hline Additional Planes Needed & $-0.0971^{* *}$ & 0.0043 & -0.0043 & -0.0858 ** & 0.0043 & -0.0038 \\
\hline \multicolumn{7}{|l|}{ Logistical Variables } \\
\hline Daily Total Airport Operations Orig. & 0.0015 & 0.0014 & 0.0001 & -0.0007 & 0.0013 & 0.0000 \\
\hline Daily Total Airport Operations Dest & $0.0077^{* *}$ & 0.0014 & 0.0003 & 0.0002 & 0.0015 & 0.0000 \\
\hline Daily Scheduled Flights & $0.0345 * *$ & 0.0011 & 0.0015 & 0.0316 ** & 0.0010 & 0.0014 \\
\hline Last Flight of Day & $-0.0590 * *$ & 0.0094 & -0.0025 & $-0.0717^{* *}$ & 0.0093 & -0.0030 \\
\hline Time01 & 0.0375 * & 0.0177 & 0.0016 & 0.0440 ** & 0.0176 & 0.0019 \\
\hline Short Flight (< 400 miles) & 0.0119 & 0.0151 & 0.0005 & -0.0167 & 0.0156 & -0.0007 \\
\hline Middle Distance (400 to 800 miles) & 0.0011 & 0.0150 & 0.0000 & $-0.0646 * *$ & 0.0156 & -0.0028 \\
\hline Labor Unrest & 0.3091 ** & 0.0190 & 0.0181 & 0.3139 ** & 0.0191 & 0.0185 \\
\hline \multicolumn{7}{|l|}{ Weather Variables } \\
\hline Minimum Temperature Origination & 0.0000 & 0.0004 & 0.0000 & -0.0002 & 0.0004 & 0.0000 \\
\hline Minimum Temperature Destination & $-0.0033 * *$ & 0.0005 & -0.0001 & $-0.0025^{* *}$ & 0.0005 & -0.0001 \\
\hline Rain Origination & 0.0022 ** & 0.0001 & 0.0001 & 0.0022 ** & 0.0001 & 0.0001 \\
\hline Rain Destination & $0.0022 * *$ & 0.0001 & 0.0001 & $0.0021^{* *}$ & 0.0001 & 0.0001 \\
\hline Frozen Precipitation Origination & 0.0122 ** & 0.0005 & 0.0005 & 0.0122 ** & 0.0005 & 0.0005 \\
\hline Frozen Precipitation Destination & $0.0106 * *$ & 0.0005 & 0.0005 & 0.0106 ** & 0.0005 & 0.0005 \\
\hline Constant & $-1.8173 * *$ & 0.0478 & & $-1.8525 * *$ & 0.0480 & \\
\hline Log Likelihood & & $-155,185$ & & & $-154,861$ & \\
\hline Pseudo R-squared & & 0.086 & & & 0.088 & \\
\hline Number of Observations & & $1,447,096$ & & & $1,447,096$ & \\
\hline
\end{tabular}

Note: Bootstrapped standard errors are reported. Regressions include carrier, day of week, month, and year dummy variables. Marginal effects are defined as the effect on the probability that the average flight is cancelled. ${ }^{*}$ and ${ }^{* *}$ indicate $5 \%$ and $1 \%$ significance levels, respectively. 
Appendix Table 1: Dummy Variables from Flight Cancellation Models $(1,2, \&$ 3)

\begin{tabular}{|c|c|c|c|c|c|c|c|c|c|}
\hline \multirow[b]{2}{*}{ Carrier } & \multicolumn{3}{|c|}{$(1)$} & \multicolumn{3}{|c|}{$(2)$} & \multicolumn{3}{|c|}{ (3) } \\
\hline & Coeff & Std. Error & Marg. Eff. & Coeff & Std. Error & Marg. Eff. & Coeff & Std. Error & Marg. Eff. \\
\hline Alaska & $-0.0805^{*}$ & 0.0353 & -0.0033 & $-0.1044^{\star \star}$ & 0.0347 & -0.0041 & $-0.0752^{*}$ & 0.0353 & -0.0031 \\
\hline Aloha & -0.4782 ** & 0.0655 & -0.0130 & $-0.4677^{* *}$ & 0.0661 & -0.0128 & $-0.4854^{* *}$ & 0.0654 & -0.0131 \\
\hline American Eagle & 0.3763 ** & 0.0298 & 0.0243 & $0.3827^{* *}$ & 0.0302 & 0.0249 & 0.3808 ** & 0.0298 & 0.0247 \\
\hline America West & 0.1185 ** & 0.0256 & 0.0059 & 0.1213 ** & 0.0257 & 0.0060 & $0.1180^{* *}$ & 0.0257 & 0.0058 \\
\hline Continental & 0.0010 & 0.0175 & 0.0000 & 0.0065 & 0.0177 & 0.0003 & 0.0059 & 0.0178 & 0.0003 \\
\hline Delta & 0.0369 * & 0.0157 & 0.0017 & 0.0490 ** & 0.0160 & 0.0022 & 0.0376 * & 0.0157 & 0.0017 \\
\hline Northwest & 0.1918 ** & 0.0208 & 0.0100 & 0.1988 ** & 0.0208 & 0.0104 & $0.1937^{* *}$ & 0.0209 & 0.0101 \\
\hline Southwest & $-0.4252^{* *}$ & 0.0230 & -0.0136 & $-0.4144^{* *}$ & 0.0231 & -0.0134 & -0.4253 ** & 0.0232 & -0.0136 \\
\hline TWA & 0.1183 ** & 0.0237 & 0.0058 & $0.1327^{* *}$ & 0.0237 & 0.0067 & 0.1218 ** & 0.0239 & 0.0060 \\
\hline United & 0.1395 ** & 0.0135 & 0.0068 & 0.1406 ** & 0.0135 & 0.0069 & $0.1431^{* *}$ & 0.0136 & 0.0070 \\
\hline US Airways & 0.1338 ** & 0.0159 & 0.0065 & 0.1432 ** & 0.0161 & 0.0071 & $0.1325^{* *}$ & 0.0160 & 0.0065 \\
\hline \multicolumn{10}{|l|}{ Month } \\
\hline January & -0.1030 ** & 0.0214 & -0.0041 & $-0.1077^{* *}$ & 0.0214 & -0.0043 & $-0.1010^{* *}$ & 0.0212 & -0.0041 \\
\hline February & -0.1669 ** & 0.0222 & -0.0063 & $-0.1710^{* *}$ & 0.0222 & -0.0065 & -0.1652 ** & 0.0221 & -0.0063 \\
\hline March & -0.2171 ** & 0.0224 & -0.0079 & $-0.2217^{* *}$ & 0.0224 & -0.0081 & -0.2162 ** & 0.0222 & -0.0079 \\
\hline April & -0.2261 ** & 0.0197 & -0.0081 & -0.2290 ** & 0.0197 & -0.0082 & $-0.2252^{* *}$ & 0.0197 & -0.0081 \\
\hline May & -0.1240 ** & 0.0160 & -0.0049 & $-0.1256^{* *}$ & 0.0162 & -0.0050 & $-0.1233^{* *}$ & 0.0160 & -0.0049 \\
\hline June & 0.0198 & 0.0182 & 0.0009 & 0.0191 & 0.0182 & 0.0009 & 0.0197 & 0.0182 & 0.0009 \\
\hline August & -0.0261 & 0.0149 & -0.0011 & -0.0259 & 0.0150 & -0.0011 & -0.0259 & 0.0149 & -0.0011 \\
\hline September & -0.1524 ** & 0.0176 & -0.0059 & -0.1524 ** & 0.0175 & -0.0059 & $-0.1515^{* *}$ & 0.0176 & -0.0058 \\
\hline October & -0.2365 ** & 0.0176 & -0.0085 & -0.2389 ** & 0.0178 & -0.0085 & $-0.2357^{* *}$ & 0.0176 & -0.0084 \\
\hline November & -0.3502 ** & 0.0220 & -0.0112 & -0.3542 ** & 0.0221 & -0.0113 & $-0.3489 * *$ & 0.0218 & -0.0112 \\
\hline Decemeber & -0.2552 ** & 0.0230 & -0.0090 & $-0.2609 * *$ & 0.0229 & -0.0091 & $-0.2535^{* *}$ & 0.0228 & -0.0089 \\
\hline \multicolumn{10}{|l|}{ Year } \\
\hline 1996 & -0.0258 & 0.0160 & -0.0011 & -0.0251 & 0.0159 & -0.0011 & -0.0253 & 0.0158 & -0.0011 \\
\hline 1997 & $-0.1307^{* *}$ & 0.0168 & -0.0053 & $-0.1303^{* *}$ & 0.0169 & -0.0053 & -0.1306 ** & 0.0168 & -0.0053 \\
\hline 1998 & -0.0062 & 0.0151 & -0.0003 & -0.0068 & 0.0152 & -0.0003 & -0.0062 & 0.0152 & -0.0003 \\
\hline 1999 & 0.0530 ** & 0.0150 & 0.0024 & 0.0520 ** & 0.0150 & 0.0024 & $0.0524^{* *}$ & 0.0150 & 0.0024 \\
\hline 2000 & $0.1377^{* *}$ & 0.0154 & 0.0066 & 0.1376 ** & 0.0154 & 0.0066 & 0.1371 ** & 0.0154 & 0.0066 \\
\hline \multicolumn{10}{|l|}{ Day of Week } \\
\hline Monday & 0.0014 & 0.0095 & 0.0001 & 0.0013 & 0.0095 & 0.0001 & 0.0014 & 0.0095 & 0.0001 \\
\hline Tuesday & 0.0066 & 0.0097 & 0.0003 & 0.0065 & 0.0097 & 0.0003 & 0.0065 & 0.0097 & 0.0003 \\
\hline Thursday & -0.0217 * & 0.0095 & -0.0009 & -0.0218 * & 0.0095 & -0.0009 & -0.0217 * & 0.0095 & -0.0009 \\
\hline Friday & $-0.0397 * *$ & 0.0105 & -0.0017 & $-0.0399 * *$ & 0.0105 & -0.0017 & -0.0398 ** & 0.0105 & -0.0017 \\
\hline Saturday & -0.0164 & 0.0114 & -0.0007 & -0.0180 & 0.0114 & -0.0008 & -0.0161 & 0.0114 & -0.0007 \\
\hline Sunday & $-0.1405^{* *}$ & 0.0104 & -0.0056 & $-0.1411^{\star *}$ & 0.0104 & -0.0056 & $-0.1402^{* *}$ & 0.0105 & -0.0055 \\
\hline
\end{tabular}

